Tonio Hölscher

\title{
Figürlicher Schmuck \\ in der griechischen Architektur \\ zwischen Dekor und Repräsentation
}

\section{Antinomien von Figur und Ornament}

Im Schmuck griechischer Tempel treffen sich Figuren und Ornamente. Damit sind zwei fundamentale Funktionen der visuellen Kunst angesprochen, die wohl für die meisten Kulturen historischer Gesellschaften konstitutiv sind: Repräsentation und Dekor. ${ }^{1}$

Repräsentation: Herstellung von Präsenz. Repräsentation im hier gemeinten Sinn ist eine Grundkategorie der visuellen Kultur: die Herstellung von Präsenz im Bild. ${ }^{2}$ Bildwerke haben die Aufgabe, bedeutungsvollen Wesen, Gegenständen und Vorgängen eine materielle Präsenz zu geben, die sie tatsächlich nicht besitzen, weil sie sich in räumlicher oder zeitlicher Distanz befinden oder rein in der Vorstellung existieren. Die wichtigsten Formen bildlicher Repräsentation sind Standbilder, in zweiter Linie Reliefs und Gemälde in großem Format. In der bildhaften materiellen Repräsentation machen sie der aktuellen Gesellschaft den kulturellen Umgang mit den dargestellten Wesen, Gegenständen und Vorgängen in sozialen Praktiken, Ritualen und Diskursen möglich. Damit beziehen sie sich referentiell auf eine - zeitlich vergangene, räumlich entfernte oder auch rein imaginierte - Realität, die eine eigene körperliche Autonomie besitzt. Diese Autonomie äußert sich im Bild darin, dass die Form so weit wie möglich frei ist von heteronomen Rahmenbedingungen und im Wesentlichen die konzeptuelle Auffassung von der Körperlichkeit der dargestellten Figuren und Gegenstände zur Erscheinung bringt.

Dekor: Steigerung von Wert. Dekor im hier gemeinten Sinn ist nicht die häufig diffamierte, oberflächliche und überflüssige Verzierung, sondern bedeutet eine Grundkategorie der ästhetischen Kultur: Schmuck als ,Pretiosierung‘, als Steigerung von kulturellem ,Wert' ${ }^{3}$ Jedes materielle Element der menschlichen Lebenskultur, das über die Erfüllung rein praktischer Funktionen hinaus eine kulturelle ,Bedeutung' hat, wird in seiner visuellen Form als Träger solcher Bedeutung ausgezeichnet. Diese Auszeichnung erhebt ,Gegenstände‘ der kulturellen Praxis im weitesten Sinn,

1 Zum Folgenden siehe vorläufig T. Hölscher 2015a, 25-51.

2 Vernant 1985, 325-338 und 339-351; Niemeyer 1996; Stähli 2002, 67-84; F. Hölscher 2014, 239-256;

T. Hölscher 2015a, 25-38.

3 Zur Definition des Ornaments siehe in diesem Band: Squire, 16-22; Grethlein, 73-77; Neer, 203-209; Barham. 
von Geräten der Religion und der Festlichkeit über Kleider und Abzeichen des sozialen Ranges bis zu Gebäuden und Anlagen des religiösen, politischen und sozialen Lebens, zu ,Bedeutung in dem doppelten Sinn von importance und meaning. Dekor in diesem Sinn des Schmückens dient der Steigerung von kulturellem Wert und Sinn. Dabei werden im Wesentlichen vier Faktoren eingesetzt:

- Wertvolle Materialien, die zugleich symbolische Bedeutung haben können, wie etwa Gold oder Purpur,

- vollendete Technik der Bearbeitung, die dem Gegenstand den Wert der Schönheit gibt,

- Ornamente, die den ästhetischen Überschuss des Gegenstands über die praktische Funktionalität darstellen,

- Ausstattung mit figürlichen Bildern, die den kulturellen ,Sinn` des Gegenstands zur Anschauung bringen.

Materialien und Technik betreffen die Gegenstände in ihrer ganzheitlichen materiellen Präsenz, Ornamente und figürliche Bilder dagegen stellen zusätzliche Elemente dar, die in einem spezifischen Verhältnis zu dem Gegenstand stehen.

Ornamente stehen in einer unlöslichen Beziehung zu ihren Gegenständen. Sie haben die Aufgabe, den kulturellen Wert des Gegenstandes durch drei visuelle Prinzipien zu steigern: durch Gliederung, Akzentuierung und Belebung. Die bekannte goldene Truhe aus dem Fürstengrab von Vergina erhält ihren Wert durch das ,königliche' Material Gold und durch hohen technischen Aufwand (Abb. 2.1). Durch ihre reichen Ornamente wird sie zum einen in ihrer Struktur und ihrem Aufbau gegliedert und definiert: durch senkrechte Pilaster an den Ecken, waagerechte Planken an den Seiten oben und unten, Reihen von Ziernägeln entlang den Kanten, rahmende Leisten am Deckel, Knäufe an den Ecken. Zum anderen erhält sie durch vegetabile und theriomorphe Motive eine bedeutungsvolle Lebendigkeit: durch Löwenfüße, Rosetten, Rankengeschlinge, Lotos und Palmetten. Auf dem Deckel erscheint als akzentuierendes Leitmotiv ein großer ,makedonischer' Stern. Wert und Bedeutung bilden eine Einheit. ${ }^{4}$

Im Bezug auf den Träger des Schmuckes werden einige Grundprinzipien des ornamentalen Schmückens deutlich. Die Rosetten und die senkrechten Reihen der Nägel entwickeln sich in der Form der Serialität, der Fries von Palmetten und Lotosblüten und die waagerechten Nägel darüber in der Form des abwechselnden symmetrischen Rapports. Der Fries von Ranken entfaltet sich aus einem zentralen Kelch von Akanthus nach dem Prinzip der Symmetrie. Die Leisten am Deckel dienen der Rahmung, die Knäufe verstärken die Rahmung durch Markierung der vorderen Ecken. Der Stern

4 Gold-Truhe Vergina: Andronikos 1989, 168-171, fig. 135-136. Zum ,makedonischen Stern“ siehe Mitropoulou 1993, 843-958; Fredricksmeyer 1997, 103; Müller 2009, 364-380. 


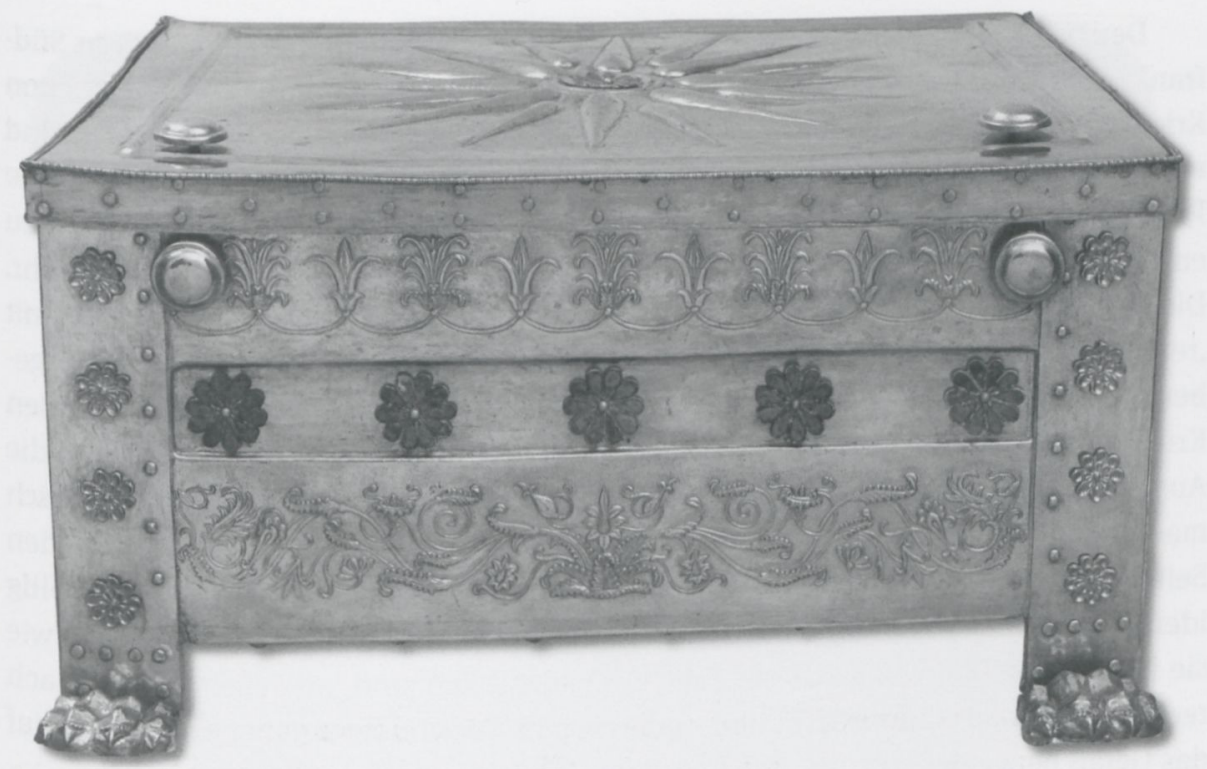

Abb. 2.1: Goldtruhe aus dem Königsgrab von Vergina, 340-320 v. Chr. Thessaloniki, Archaiologikon Mouseion.

wirkt durch seine gerahmte Zentralität. In allen diesen Formen sind Ornamente auf ihren Träger bezogen: Es gibt kein autonomes Ornament. ${ }^{5}$

Figürlicher Schmuck hat die Aufgabe, den kulturellen Wert des Gegenstandes durch bildliche Darstellung von Figuren zu steigern und mit deren komplexen kulturellen Bedeutungen zu bereichern. Damit wird aber ein grundsätzlicher Konflikt zwischen Repräsentation und Dekor eingeführt. Denn die Bilder haben zunächst andere Aufgaben, mit anderen Erfordernissen als denen des Schmückens: die Präsent-Machung von Gestalten und Vorgängen. Während die Bilder als Repräsentation von Gestalten, Handlungen und Vorgängen eine genuine Autonomie besitzen, werden sie gleichzeitig heteronom durch die Strukturen der zu schmückenden Gegenstände bestimmt. Diesen Vorgaben der Bildträger ordnen die Figuren der Bilder sich aber nicht ohne Widerstand unter: Die Repräsentation von Figuren und Handlungen lässt sich nicht eo ipso mit ornamentalen Prinzipien wie Serialität, Rapport, Symmetrie, Gerahmtheit und Zentralität vereinbaren. Dieser latente Konflikt zwischen Ornament und Figur, die Heteronomie von Dekor und Repräsentation, ist im figürlichen Schmuck von Gegenständen und Bauwerken in immer wieder neuer Form und mit vielfach variierenden Prioritäten ausgetragen worden.

$5 \mathrm{Zu}$ Grundprinzipien des Ornaments siehe in diesem Band insbesondere: Grethlein, 73-77; Haug, 97-101; Dietrich, 172-179; Barham, bes. 281-289. 
Der berühmte Bronzekrater aus dem Grab einer fürstlichen Frau bei Vix in Südfrankreich ${ }^{6}$ trägt um den Hals einen Relieffries mit der Darstellung eines Zuges von Kriegern, in dem sich ein Wagen mit vier Pferden sowie einem Lenker mit Helm und ein vornehmer Krieger in voller Rüstung zu Fuß abwechseln (Abb. 2.2). Ob eine rituelle Prozession oder ein feierlicher Auszug in den Krieg geschildert wird, ist schwer zu entscheiden; im Grundkonzept unterscheiden sich die beiden Themen nicht sehr. Die Pferde sind in stark unterschiedlichen Haltungen dargestellt und scheinen damit ,realistisch“ die lebendige Vielfalt der Bewegungen bei dem Vorgang wiederzugeben. Doch die einzelnen Gespanne, mitsamt den Lenkern und den nachfolgenden Kriegern, sind in völlig identischer Form aufgereiht. Der ,Realität‘ ist wiederum die Ausrichtung des Zuges nach rechts geschuldet, die eine axiale Symmetrie unmöglich macht. Doch dabei steht ein Krieger in der Mittelachse des Gefäßes, und auf beiden Seiten sind bis zu den Henkeln je zwei Gespanne mit schreitenden Kriegern in völlig identischen Abständen zu sehen. Die Prozession ist somit ohne alle Variationen, wie sie in anderen Bildwerken dieser Zeit durchaus üblich sind, als regelmäßiger, nach rechts gerichteter Rapport gestaltet und in seiner Anordnung streng ,ornamental ' auf das Gefäß bezogen.

Die Ansätze der Volutenhenkel sind mit Gorgonen geschmückt, deren Körper in extremer Weise für ihre ornamentale Funktion verformt sind. Die Figuren sind mit der frontalen Gorgonenfratze und symmetrisch angewinkelten Armen nach außen gerichtet; ihr Körper ist so beschnitten, dass unmittelbar unter der Brust mit dem kurzen Wams zwei Schlangen-,Beine' herauswachsen, die sich zu beiden Seiten zur technischen Verstärkung der Befestigung auf die Schulter des Gefäßes legen. Zwei weitere Schlangen, deren Ansatz an ihrem Körper nicht deutlich ist, winden sich um ihre Oberarme und richten sich symmetrisch zu Seiten ihres Gesichts auf. Im Henkel sind Löwen in unorganischer Haltung, mit aufgerichtetem Körper und zurückgedrehtem Kopf, in die Zwickel des Volutenhenkels eingepasst. Monster, Tiere, Henkel und Gefäßkörper vereinen sich zu einem Gebilde von höchster ornamentaler Künstlichkeit. $^{7}$

Im Zentrum des Deckels steht eine weibliche Figur, in vornehmen Kleidern, durch einen Schleier auf dem Kopf, Opferkanne in der linken und Opferschale in der vorgestreckten rechten Hand (beide zu ergänzen) als Braut ausgezeichnet, die sich auf die Krieger bezieht. Mit ihrem stark säulenhaften Wuchs besitzt sie fast die ,ornamentale‘

6 Rolley 2003, 77-143.

7 Der Konflikt zwischen Repräsentation und Ornament reicht bis in die Details der Figuren. Das kann hier nicht weiter verfolgt werden. Er wird pointiert deutlich am Bild der Gorgo, etwa am Krater von Vix: Die repräsentierte (vorgestellte) Realität ist eine Fratze von extremer Hässlichkeit, die ,künstlerische“ Gestaltung ist von exquisiter Perfektion und Schönheit: Die Stirnlocken der Gorgonen sind ebenso brillant ornamental gestaltet wie die Ornamentbänder der Henkel, siehe Rolley 2003, Taf. 98-99. Die Hässlichkeit bezieht sich auf das Thema, die Schönheit auf das Werk, als Gegenstand der Lebenskultur bzw. des religiösen Kults. 


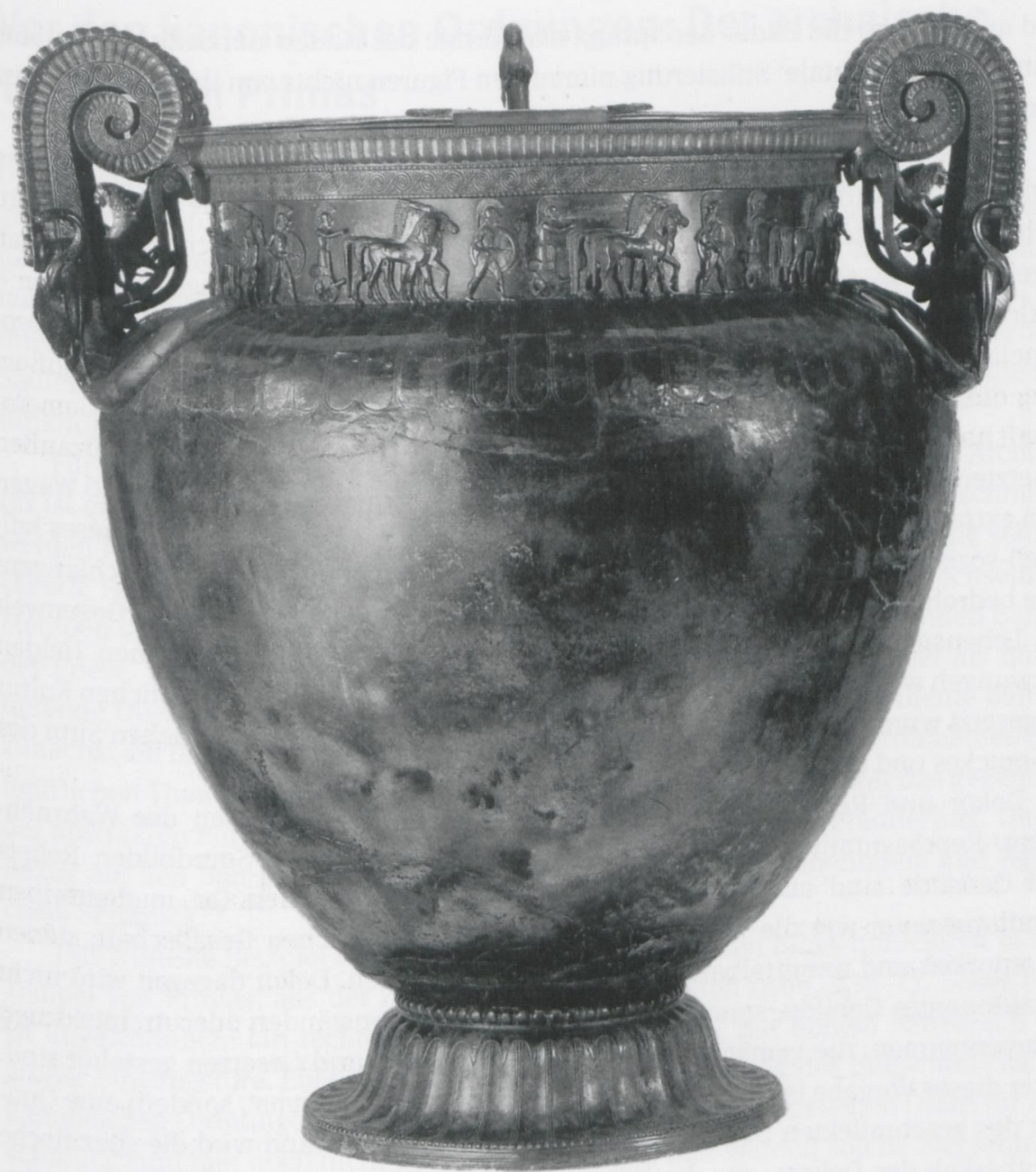

Abb. 2.2: Bronzekrater aus dem Fürstinnengrab von Vix, Ende 6. Jh. v. Chr. Châtillon-sur-Seine, Musée du Pays Châtillonnais.

Form eines länglichen Knaufes. Dem gegenüber bedingt die Realität des menschlichen Körpers und insbesondere das Motiv von Arm und Hand eine Abweichung von der ,ornamentalen‘ Rundung eines Knaufes.

Am Krater von Vix ist der Konflikt zwischen den abstrakten Prinzipien des Ornaments und der körperlichen Realität der Figuren in einem besonders hohen Maß im Sinn des Ornaments gelöst. Gleichwohl aber bewahren die Figuren ihre körperliche Präsenz, Kraft und Wirkung. Insbesondere die Gorgonen und Löwen sind gewiss als bildhafter Schutz des Gefäßes vor Beschädigung und seiner Nutzer vor schädlichen Wirkungen des Weines angebracht - in demselben Sinn wie an Quellhäusern die Wasserspeier in Form eines Löwenkopfes als ,Quellwächter (krenophylax) bezeichnet 
und auf Gräbern die Bilder der Sphinx als ,Hunde des Hades‘ um Schutz angerufen wurden. ,Ornamentale‘ Stilisierung nimmt den Figuren nichts von ihren, lebendigen` Wirkung. ${ }^{8}$

Insgesamt fügt sich der Schmuck des Kraters von Vix zu einem konzentrischen Konzept einer kulturellen Ordnung zusammen. Im Zentrum steht die Frau als Braut, nicht nur weil der Krater als prunkvolle Gabe einer jungen Fürstin mit ins Grab gegeben wurde, sondern allgemein, weil in den archaischen Adelsgesellschaften in Griechenland wohl ähnlich wie im keltischen Norden - die Frau ihren konzeptionellen wie realen Ort im inneren Bereich von Haus und Herd hatte. Der äußere Ring dieser Ordnung wird von der Prozession der Krieger gebildet, die die Gemeinschaft nach außen verteidigten und auch mit ihren Ritualen den Bereich des Draußen besetzten: So zogen die Adeligen von Eretria in einer großen Prozession auf 60 Wagen zum extraurbanen Heiligtum der Artemis Amarynthia. Den äußeren Rand dieses kulturell-sozialen Konzepts markieren schließlich die Gorgonen, Löwen und Schlangen: jene bedrohlichen Monster und Bestien der Wildnis, die die konzeptuelle Gegenwelt zur Lebensordnung der Menschen bildeten, von Göttern und mythischen Helden bezwungen wurden und schließlich symbolisch zum Schutz der menschlichen Kultur eingesetzt wurden. Die Bilderwelt des Kraters ist ein Kosmos im zweifachen Sinn des Schmuckes und der (Lebens-)Ordnung. ${ }^{9}$

Dekor und Repräsentation bedingen unterschiedliche Formen der Wahrnehmung. Repräsentative Bildwerke, insbesondere großformatige Standbilder, Reliefs und Gemälde sind auf Interaktion angelegt. Sie haben ihren Ort in demselben Handlungsraum wie die lebenden Akteure der menschlichen Gesellschaft, denen sie autonom und unmittelbar sichtbar gegenüber stehen. Dekor dagegen wird nicht als autonomes Gebilde, sondern als Element von Gegenständen oder Architekturen wahrgenommen, die gemäß ihren eigenen Funktionen und Gesetzen gestaltet sind. Unter dieser Vorgabe ist Dekor zunächst kein autonomes ,Thema', sondern eine Qualität des geschmückten Gegenstandes. Von diesem Gegenstand wird die spezifische Sichtbarkeit des Dekors, aus der Nähe oder in der Distanz, mit größerer oder geringerer Aufmerksamkeit, mit dem Blick auf das Einzelne oder als Wahrnehmung eines allgemeinen Eindrucks, bestimmt.

Die hier kurz bezeichneten Probleme und Konflikte zwischen Figur und Ornament zeigen sich mit besonderer Schärfe im figürlichen Schmuck von Architektur.

8 Zur schützenden Funktion von Löwen, Gorgonen, Sphingen siehe F. Hölscher 1972, 59-66.

9 Konzentrische Struktur der griechischen Polis-Welt: T. Hölscher 2000; T. Hölscher 2015a, 21-22. Prozession mit 60 Wagen von Eretria zum Heiligtum der Artemis Amarynthia: Strabo 10, 1, 10. Wilde Randzonen (eschatiä) und wilde Tiere/Monster: T. Hölscher 1999; Winkler-Horaček 2015, bes. 371-394. 


\section{Vor den kanonischen Ordnungen: Der archaische Tempel von Prinias}

Die Ausstattung von Architektur mit figürlichem ,Decor‘ steht zunächst unter der grundsätzlichen Voraussetzung, dass bereits die Bauwerke als solche durch und durch nach ,decorativen“ Konzepten gestaltet sind: in rechteckigen oder runden Grundformen, in Proportionen von Länge, Höhe und Tiefe, auf emporhebenden Stufen, mit intentional (vielfach axial) gesetzten Türen und Fenstern, rhythmisch gesetzten Säulen, deckenden Gebälken und einem auf Ansicht gestalteten Dach. Schon die rein funktionalen Elemente erhalten eine ,decorative‘ Form.

Für die Bewertung des figürlichen ,Decors‘ griechischer und römischer Architektur ist entscheidend, dass alle Elemente der Bauwerke, die mit Figuren geschmückt werden konnten, auch ohne Figuren eingesetzt wurden und als solche eine schmückende Funktion hatten: in der dorischen Ordnung die Reihen von abwechselnden Metopen und Triglyphen, in der ionischen Ordnung die zusammenfassenden Bänder der Friese, in verschiedenen Ordnungen die flachen dreieckigen Giebel als nobilitierende Überhöhung der Fassade. Die dekorative Wirkung dieser Elemente beruhte zunächst auf ihrer ,leeren` abstrakten Form. Wenn sie zusätzlich mit Ornamenten oder figürlichen Themen in Malerei oder Relief geschmückt wurden, so war das gegenüber der abstrakten Form eine Bereicherung, aber keine essentielle Veränderung. Und in Bezug auf den Bau gab es keinen Unterschied zwischen ornamentalem und figürlichem Schmuck. ${ }^{10}$

Das früheste Beispiel eines umfangreichen, komplexen Bildschmucks ist in dem Tempel von Prinias, aus der Zeit um 630 v. Chr. erhalten (Abb. 2.3a-c). Seine Form ist ungewöhnlich: ein rechteckiges Haus, ohne Säulen, mit hohem Satteldach und großer Eingangstüre. Ebenso unkanonisch ist die Platzierung des Bildschmucks: Die kanonischen Ordnungen der Tempelarchitektur mit ihren festgelegten Orten für Bildschmuck sind hier noch nicht in Sicht. Doch schon hier werden solche Elemente des Bauwerks, die bereits als solche ,decorative ' Funktion haben, zusätzlich mit figürlichem Schmuck hervorgehoben: Orthostaten der Wand, Gewände der Türe, Rahmen der Lichtöffnung. ${ }^{11}$

In den Bildern ist, entsprechend dem Krater von Vix, ein Konzept einer Lebensordnung zu erkennen, das in innere und äußere ,soziale‘ Bereiche gegliedert ist. Wie an keinem anderen griechischen Kultbau ist der figürliche Schmuck der Architektur ,ornamental‘ eingefügt - zugleich aber wird hier in besonderem Maß deutlich, dass die Ordnung des Schmuckes eine Ordnung der Bildthemen ist.

10 Zum schmückenden Charakter von Metopen-Triglyphen, Friesen und Giebeln siehe Marconi 2004. 11 Zum Tempel von Prinias siehe Beyer 1976, 21-38; D’Acunto 1995, 15-55; Marinatos 2000, 67-78; T. Hölscher 2015a, 89-91. 


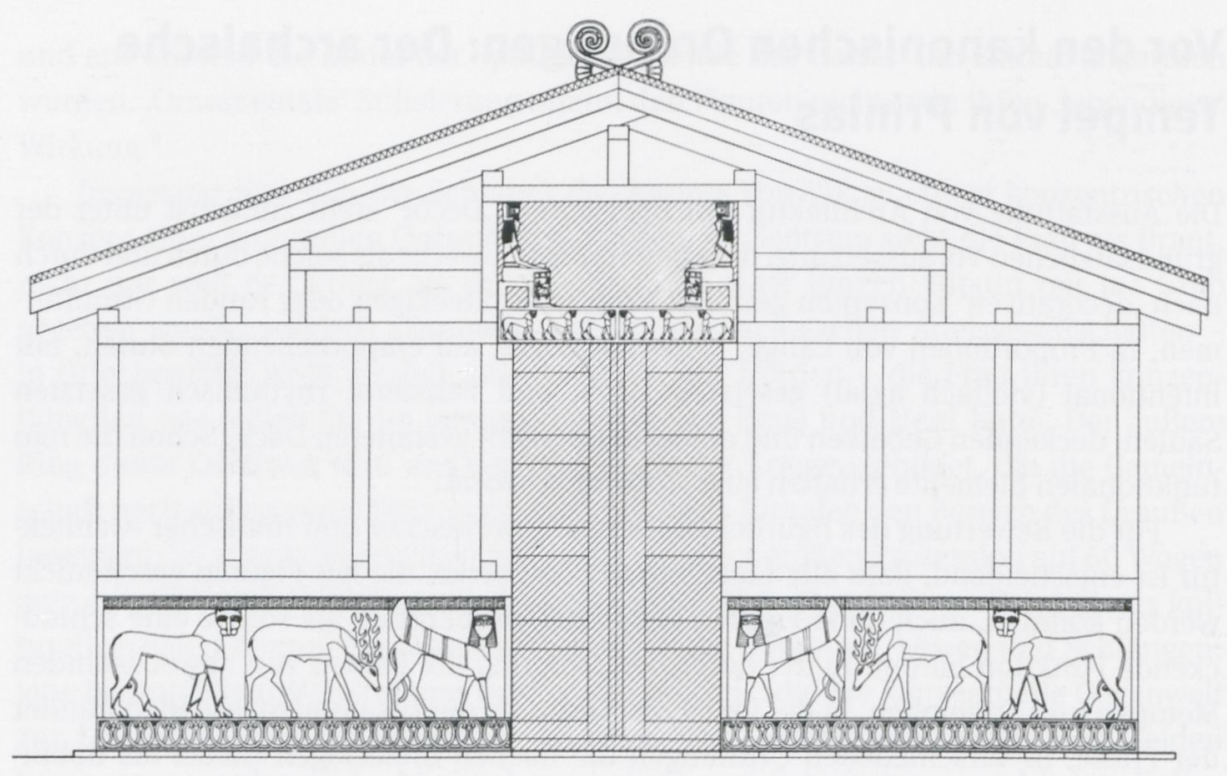

Abb. 2.3a-c: Archaischer Tempel (AS) von Prinias, Rekonstruktion des Bildschmucks. Um 630 v. Chr.

Im Inneren des Baues ist wohl ein Kultbild der Gottheit anzunehmen, die wohl für die Gemeinschaft von Prinias ,poliadische“ Bedeutung gehabt hat. Der Bildschmuck des gesamten Tempels lässt an eine weibliche Göttin denken, am ehesten Artemis. Die große Eingangstüre wird von Frauen beherrscht, die dem Haus der Gottheit zugeordnet sind, offenbar in einer Hierarchie von drei Altersstufen: nackte Mädchen stehend, bekleidete junge Frauen stehend, bekleidete Frauen thronend, jeweils in symmetrischer Verdoppelung. Sie sind zuletzt sämtlich allgemein als weibliche Gottheiten gedeutet worden, doch das ist unwahrscheinlich: Wenn dieselbe Göttin gemeint wäre, so wäre die Vervielfältigung in drei unterschiedlichen Aspekten höchst ungewöhnlich; wenn verschiedene göttliche Wesen vereinigt wären, so wäre auch eine derart differenzierte Abstufung ohne Vergleich. Tatsächlich scheinen mindestens zum Teil menschliche Wesen gemeint zu sein. ${ }^{12}$

An den Innenseiten der Türpfeiler erscheinen nackte Mädchen, die offenbar das Stadium der Initiation repräsentieren. Die zahlreichen weiblichen Figuren mit nacktem Körper aus dem 8. und 7. Jahrhundert v. Chr., vor allem Statuetten und Reliefs aus Terrakotta, Bronze und anderen Materialien, sind ikonographisch zumeist nicht genauer charakterisiert; aber mit Sicherheit stellen sie nicht alle „die nackte Göttin“ dar. Das gilt insbesondere für Gruppen von zwei bis zu fünf Figuren, die zu Recht als junge Mädchen angesprochen wurden. Dabei ist nicht nur an spezielle Kultdienerinnen zu denken, sondern allgemein an die weibliche Jugend der Oberschicht. In

12 Dieselbe Göttin in verschiedenen Aspekten: Marinatos 2000. 
b
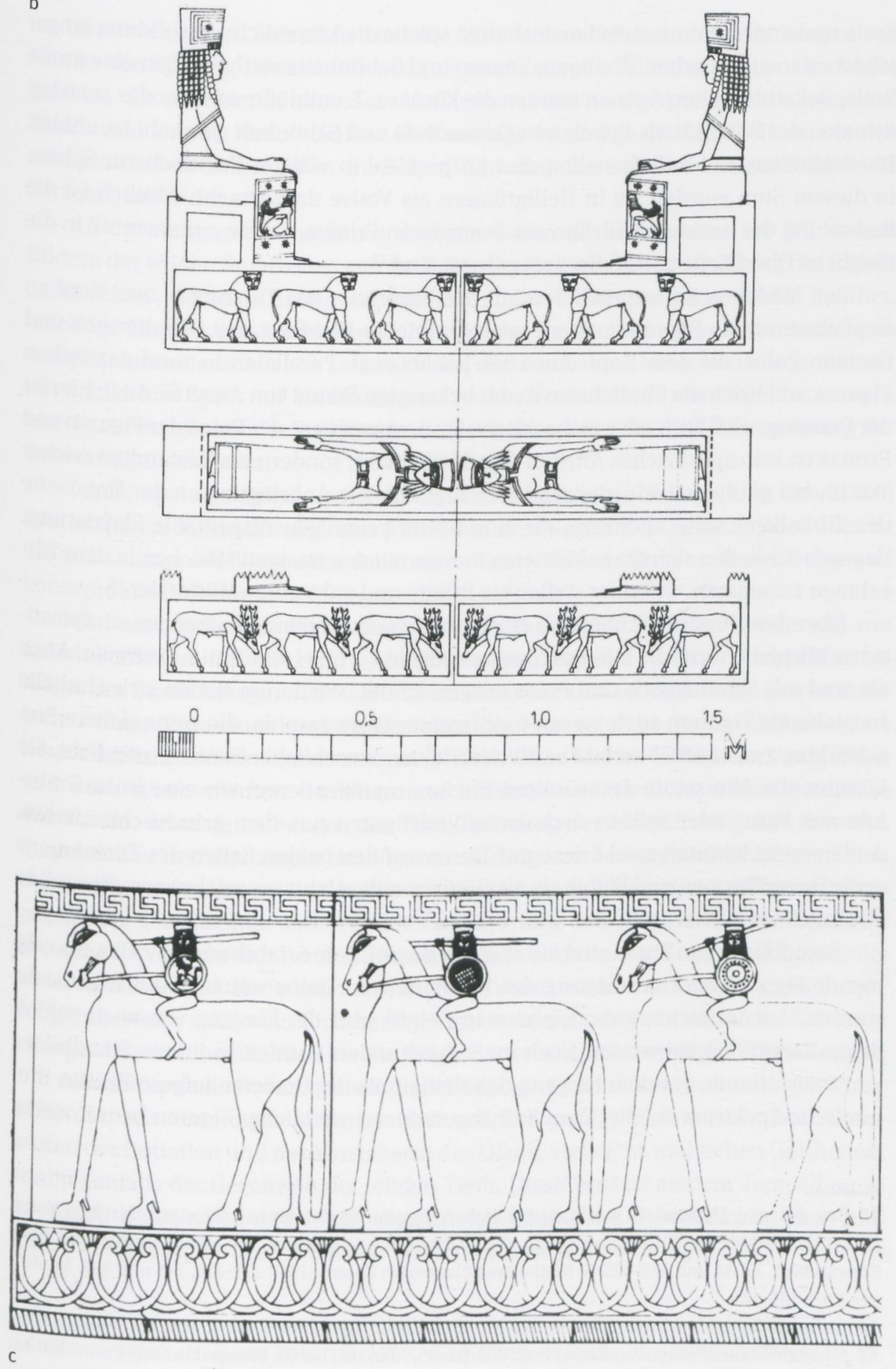
Kreta und anderen dorischen Landschaften spielte die körperliche Ausbildung junger Mädchen in athletischen Übungen, Tänzen und Schönheitswettbewerben eine große Rolle. Bei athletischen Agonen wurden die Körper z. T. entblößt; wie weit das auch bei Ritualen der Pubertät als Erweis von Gesundheit und Schönheit geschah, ist unklar. Die Statuetten und Reliefs stellen dies Körperideal in völliger Nacktheit zur Schau; in diesem Sinn wurden sie in Heiligtümern als Votive dargebracht. Ähnlich ist die Bedeutung der nackten Mädchen am Tempel von Prinias, wo sie ,ornamental ‘ in die längliche Oberfläche des Pfeilers eingepasst sind. ${ }^{13}$

Den Mädchen korrespondieren auf der Unterseite des Türsturzes zwei Kopf an Kopf angeordnete Figuren junger Frauen in reicher Kleidung, mit Schultertuch und flachem Polos auf dem Kopf. Auch sie haben enge Parallelen in rundplastischen Figuren, auf Kreta am ähnlichsten in der bekannten Statue von Auxerre. Auch hier ist die Deutung, ob Göttin oder menschliche Frau, umstritten; der Polos der Figuren von Prinias ist kein spezifisches Attribut von Göttlichkeit, sondern ein Bestandteil reicher Tracht, bei göttlichen wie menschlichen Figuren. Die Anbringung an der Unterseite des Türbalkens wäre allerdings für eine Göttin keine sehr respektable Platzierung, dagegen schließen sich die bekleideten Frauen mit den nackten Mädchen in dem Türrahmen zusammen. Offenbar stellen sie Bräute und zukünftige Mütter dar. ${ }^{14}$

Über dem Türsturz schließlich erscheinen zwei thronende Frauen, in rundplastischer Körperlichkeit, die einander zugewandt eine große Lichtöffnung rahmen. Auch sie sind mit Schultertuch und Polos ausgezeichnet. Wiederum finden sich ähnliche freistehende Figuren, auch sie mit indifferenter Ikonographie, die keine sichere Entscheidung zwischen Götterbild und Votivbildern menschlicher Frauen ermöglicht. Sie könnten die Altersstufe der würdigen Ehefrau repräsentieren, wie eine frühe Grabstele aus Paros oder spätere archaische Votivfiguren aus dem griechischen Osten. Andererseits könnten zwei Friese mit Tieren auf den beiden Seiten des Türrahmens unter ihren Thronen und Füßen sie als Göttinnen der Natur auszeichnen: außen wilde Panther, innen Hirsche. ${ }^{15}$

Standbilder von Koren sind kürzlich für das mittlere 6. Jahrhundert v. Chr. als rahmende Figuren am Nordeingang des Heraions von Samos wahrscheinlich gemacht worden. Am Schatzhaus von Siphnos in Delphi wird der Eingang von reich gekleideten Karyatiden eingefasst. Noch im 3. Jahrhundert wurden in Priene Standbilder von Priesterinnen vor dem Eingang des Heiligtums der Demeter aufgestellt. Das thematische Spektrum solcher dem Kult zugeordneter weiblicher Figuren bedarf weite-

13 Das gesamte Material zu nackten weiblichen Figuren in der archaischen griechischen Kunst (außer den Reliefs von Prinias) bei Böhm 1990. Athletische Ausbildung von Mädchen: Arrigoni 1985; Stewart 1997, 29-34 und 108-129. Schönheitswettbewerbe: Calame 1977, 223-224, 345 und 447; Kreilinger 2007, 186-189.

14 Stehende bekleidete Frauenfiguren aus Kreta: Bol 2002, 83-90, Abb. 160 und 162 (G. Kaminski). 15 Sitzende Frauenfiguren aus Kreta: Bol 2002, 92-95, Abb. 167-169 (G. Kaminski). Stele Paros: Barlou 2014, 17, Taf. 1. 
rer Untersuchungen. Jedenfalls sind die Mädchen und Frauen, die am Tempel von Prinias den Eingang rahmen, offenbar als empfangende Teilnehmerinnen des Kultes gemeint. Sie sind hier in extremem Maß ,ornamental' in die Architektur eingepasst. Gleichwohl kommt ihren offenbar eine analoge lebendige Wirksamkeit zu wie den rundplastischen Stützfiguren in Delphi und den frei stehenden Standbildern in Samos(?) und Priene. ${ }^{16}$

An der Außenseite des Baues dagegen waren die Orthostaten der Wände mit Bildern der wilden Randzonen geschmückt, die die konzeptuelle Ordnung der frühen ,städtischen' Gemeinschaften umgaben: an der Frontseite bedrohliche Panther, mächtige Hirsche und monströse Sphingen, das ganze Spektrum der animalischen Antipoden zur menschlichen Lebenskultur; und von beiden Langseiten junge Männer mit Schild und Speer auf hochbeinigen Pferden heranreitend: die Blüte der vornehmen Familien, die auf der Jagd und im heldenhaften Krieg für die Lebensordnung der Gemeinschaft kämpften.

Der Tempel von Prinias wird durch seinen figürlichen Schmuck zu einem Bau von höchstem kulturell-religiösen ,Wert‘, ein agalma für die Gottheit. Auch hier ist die Ausstattung mit Bildwerken ein Kosmos im zweifachen Sinn: kostbarer Dekor und konzeptuelle Ordnung.

Als Dekor stellen die Bildwerke eine Pretiosierung im Sinne der Steigerung von kulturellem Wert dar. Sie sind in besonders starkem Maß auf die Architektur als Träger der Bilder bezogen. Die dadurch entstehende Heteronomie zeigt sich in zweifacher Weise. Zum einen sind die Gestalten der Figuren nicht nur durch ihre eigene Körperlichkeit, sondern sehr stark auch durch ihren Ort im Gefüge der Architektur und ihrer symmetrischen Grundstruktur bestimmt: relativ hohe Orthostatenfriese der Wände, schmale Laibungen der Türe, Rahmung der Lichtöffnung. Die Formate, Proportionen und Haltungen der Figuren sind rigoros den architektonischen Orten angepasst; an der Unterseite des Türsturzes sind die Frauen sogar antithetisch Kopf gegen Kopf gestellt. Zum anderen bietet die Ordnung der Bilder sich dem Blick nicht in einfacher Ansicht dar: An den Türpfeilern wie auch an der Unter- und Innenseite des Türsturzes sind die Reliefs nur mit Mühe wahrzunehmen. Schmuck, als Steigerung von ,Wert', setzt zwar Sichtbarkeit voraus, besitzt aber als kulturelle Kategorie eine gewisse Autonomie, die über die möglichst deutliche Ansichtigkeit hinausreicht.

Als konzeptuelle Ordnung stellen die Bilder eine soziale und kulturelle ,Welt‘ der Polis dar: mit den jungen Männern in ritterlicher aretē, den Frauen in den Altersstufen der Initiation und der Vorsteherin des Oikos, vielleicht weiblichen Gottheiten, und jedenfalls der Gegenwelt der wilden Tiere. Diese Welt ist an dem Tempelbau so geordnet, dass Außen und Innen die sozialen Räume der Geschlechter, Oben und Unten die Hierarchie der weiblichen Wesen zur Anschauung bringen.

16 Heraion, Samos: Duplouy 2006, 197-203. Schatzhaus der Siphnier, Delphi: Schmidt 1982, 72-78. Demeter-Heiligtum, Priene: Eule 2001, 121. 
Man kann sich vorstellen, dass vor und in dem Tempel die religiösen Rituale der städtischen Gemeinschaft in visuell gestalteter Form vollzogen wurden. Die Ordnungen der Bilder und der realen Gesellschaft ergänzten sich wechselseitig.

\section{Kanonischer Bauschmuck: Der klassische Tempel des Zeus in Olympia}

Der Zeus-Tempel von Olympia ist ein fortgeschrittenes Beispiel einer kanonischen Bauordnung mit komplexem Bildschmuck. Vorausgegangen waren dorische Tempel in Unteritalien und Sizilien, ebenso ionische und dorische Schatzhäuser in Delphi und Olympia. Der Zeus-Tempel macht besonders eklatant eine Eigenschaft aller archaischen und klassischen griechischen Bauskulptur deutlich: ihre extrem schlechte Sichtbarkeit. ${ }^{17}$

Bereits früher wurde hervorgehoben, dass die Platzierung von figürlichem Schmuck an griechischen Sakralbauten denkbar ungünstig für die Wahrnehmung durch die Betrachter ist. Es ist, zumindest in diesem Zusammenhang, wenig sinnvoll, darüber zu streiten, wie viel antike Betrachter in den figürlich gestalteten Metopen, Friesen, Giebeln und Akroteren haben sehen können. Entscheidend ist, dass die Platzierung dieser Bildträger zweifellos nicht mit dem Ziel bestmöglicher Sichtbarkeit festgesetzt wurde: Sie schmücken Gebälk und Dach in großer Höhe, bieten sich dem Blick nur in großer Distanz, und bei verringerter Distanz in zunehmend steilem Winkel dar. ${ }^{18}$

Durchweg handelt es sich um architektonische Elemente, die nicht zum tektonischen Gerüst des Bauwerks gehören: Die Metopen als Leerstellen zwischen den ,konstruktiven' Triglyphen, die Friese als Schmuckband über dem tragenden Architrav, die Giebel als leere Dreiecke zwischen Gebälk und Dach, die Akrotere als Aufsätze über dem Dach. Als nicht-konstruktive Elemente haben sie im Kontext der Architektur zunächst ornamentale Wirkung: die Metopen als serieller Rapport, die Friese als Band, die Giebel als symmetrische Bekrönung, die Akrotere als Akzente über First und Ecken. Aufgrund dieser ornamentalen Grundfunktion können sie - im Gegensatz zu den konstruktiven Elementen der Stufen, Wände, Säulen und Gebälke - mit zusätzlichen Ornamenten geschmückt werden: vielfach in Malerei, und darum nicht mehr erhalten.

$17 \mathrm{Zu}$ dem Skulpturenschmuck des Zeus-Tempels von Olympia siehe bes.: Herrmann 1987; Simon 1968; Kyrieleis 2012/2013. Die Einwände von Patay-Horváth 2007 gegen die Rekonstruktion des Ostgiebels und seine Deutung durch Pausanias überzeugen mich nicht, siehe Kyrieleis 2012/2013, 67 Anm. 37. Dazu zuletzt Patay-Horváth 2015, 2-6, 94-97 und 188-200; dagegen T. Hölscher in PatayHorváth 2015, 90-93.

18 Problematische Sichtbarkeit: T. Hölscher 2009. Entsprechend zum Parthenon siehe Marconi 2009. 
Wenn seit dem 6. Jahrhundert v. Chr. Metopen, Friese, Giebel und Akrotere auch in Form von figürlichen Bildern geschmückt wurden, so war dabei offenbar nicht primär die Absicht maßgebend, bildliche,Botschaften` in möglichst zugänglicher Ansicht für die Betrachter zu präsentieren, sondern vorgegebene ,ornamentale‘ Felder mit einem besonders reichen und bedeutungsvollen Schmuck zu füllen. Dafür waren aber diese Felder auch in ihren Formaten schlecht geeignet: Die langen Serien quadratischer Metopen eigneten sich nur für eng begrenzte Kompositionen von wenigen Figuren; komplexere Themen konnten nur aus solchen immer gleichen Teil-Bildern zusammengesetzt werden. Die langen und schmalen Formate der Friese ließen fast nur Themen von grundsätzlich sequentieller Form zu. Die Giebel mit ihren flachen dreieckigen Rahmen zwangen zu zentralen Kompositionen mit höchst artifiziellen Bewegungen und Haltungen zu den Ecken hin. Die Akrotere setzten mit ihrer Funktion und Platzierung am Bau der Wahl der Themen enge Grenzen.

Die Macht der ,ornamentalen` Vorgaben ist am Zeus-Tempel von Olympia besonders deutlich zu erkennen. In beiden Giebeln wie in den beiden Reihen von Metopen werden die mythischen Vorgänge in einer Form vor Augen geführt, die in hohem Maß von den strukturellen Vorgaben der Architektur geprägt ist und die figürliche Repräsentation in einer Form der ornamentalen Lebendigkeit entfaltet.

Der Ostgiebel mit der Konstellation der Protagonisten Oinomaos und Pelops vor der Wettfahrt um die Herrschaft in Olympia ist in seiner symmetrischen Grundstruktur oft analysiert worden (Abb. 2.4). Die Rekonstruktion der Komposition ist zuletzt von Helmut Kyrieleis noch einmal mit guten Gründen im Sinne von Reinhard Kekulé von Stradonitz, Hans-Volkmar Herrmann und Erika Simon bestätigt worden; doch die in den erhaltenen Figuren inhärente Kraft der formalen Grundprinzipien zeigt sich darin, dass auch andere Rekonstruktionen durchweg zu Kompositionen von dynamisch variierter Symmetrie führen. Die antithetische Ordnung der Gestalten ist von großer Prägnanz: Zeus als Mittelachse, gerahmt von den beiden Paaren König/ Königin und Freier/Braut, sodann die beiden Gespanne, anschließend die beiden Paare von hockendem Sehern und je einer weiteren kauernden Gestalt, in den Zwickeln die gelagerten Flussgötter. Um dieser Symmetrie willen wird die mythische Situation in einer Form geschildert, die in verschiedener Hinsicht nicht von dem ,realen` Vorgang geprägt ist. Um nur wenige Beispiele zu nennen: Die antithetische Stellung der Gespanne lässt nichts davon erkennen, dass beide in dieselbe Richtung abfahren werden; der Pferdeknecht vor dem Gespann des Pelops wird von einer Dienerin der Sterope ausgeglichen, die unmittelbar vor den Pferden des Oinomaos einen wenig natürlichen Platz hat; das Lagern der Flussgötter entspricht nicht der üblichen Ikonographie von Flussgöttern in dieser Zeit, sondern ist von der Form des Giebels diktiert.

Die Volumina und Silhouetten dieser Komposition bilden ein abstraktes Muster: im Zentrum fünf senkrecht aufstrebende Körper, dann die waagerechten Trapeze der Gespanne, in den Zwickeln die gestauchten Leiber der hockenden Figuren, und in den Zwickeln die Liegenden mit ihren fließenden Gestalten. Daraus ergibt sich eine 
dekorative Struktur, die im Prinzip den Rankenornamenten von kleineren Giebeln entspricht. Vergleichbar sind etwa die Kompositionen in den Giebeln der kleineren Sarkophage aus dem Grab des Alexandersarkophags von Sidon (Abb. 2.5): Dort steht im Zentrum eine große Blüte, vertikal aufgerichtet und frontal zum Betrachter gewendet, wie die fünf zentralen Figuren in Olympia. Nach den Seiten entwickeln sich die Kompositionen grundsätzlich horizontal: die Ranken zunächst abwärts eingerollt, entsprechend den Gespannen mit gesenkten Schweifen; dann aufwärts zurückgerollt, entsprechend links dem Lenker, rechts dem ersten Seher; dann in die Zwickel auseinanderfließend, wie die Flussgötter. ${ }^{19}$

Dies Prinzip der antithetischen Symmetrie ist allerdings in der Komposition von Olympia in komplexer Weise vielfältig aufgebrochen. Helmut Kyrieleis hat in einer eindringlichen Studie die komplexe Körper- und Gebärdensprache des Giebels untersucht und gezeigt, in welchem Maß sie die gesamte Auffassung des ,realen‘ Mythos prägt. Zeus wendet und öffnet sich zu seiner rechten Seite, als Zeichen seiner Zustimmung zu Pelops‘ Sieg. Die beiden Paare sind durch unterschiedliche Tracht als älteres Königspaar und heldenhaftes Brautpaar charakterisiert. In ihrer Haltung kommen die verschiedenen psychischen Zustände zum Ausdruck: Oinomaos erscheint in statischer Pose der herrscherlichen Arroganz, die rechte Hand abwartend in die Hüfte gestützt, Sterope in energieloser Haltung, mit sich verschließenden Gebärden der Arme, die die Vorahnung des Unglücks anzeigen, beide von Zeus abgewandt und dem Verderben entgegenblickend. Ihnen gegenüber stehen Pelops mit dem beweglichen Körper des jungen Helden, den Kopf in Bescheidenheit und Selbstkontrolle gesenkt, und Hippodameia in der stattlichen Erscheinung als glanzvolle Braut, mit Gebärden der sich öffnenden Anmut, beide in liebender Zuneigung einander zugewandt. Anschließend bilden die beiden Gespanne zwei gleich gewichtige Blöcke, doch Pelops` Gespann wird von zwei Dienern für den baldigen Start vorbereitet, das später startende Gespann des Oinomaos ist noch ohne Personal. Sehr unterschiedlich sind sodann die Seher, nach alter olympischer Tradition auf dem Boden sitzend, die die unterschiedliche Zukunft der Protagonisten antizipieren: rechts ein Greis, Amythaon oder Klytios, der mit bedrückter Miene und einer Gebärde der Sorge das Unglück des Königs voraussieht; daneben ein jüngerer, wohl Melampous, in passiver Haltung und mit gesenkt abgewandtem Kopf; links dagegen Iamos, in kräftiger Männlichkeit, der mit erhobenem Kopf zuversichtlich zu Zeus und den von ihm beschützten Brautleuten Pelops und Hippodameia schaut. Die Flussgötter sind in ihren Körpern entsprechend ihrem natürlichen Charakter differenziert: links der ältere Alphaios als ruhiger kraftvoller Strom, rechts der jüngere Kladeos als ungestüm vorandrängender Bach.

19 Sarkophage Sidon: Möbius 1929, Taf. 25a-b; Graeve 1970, Taf. 2.2 und 3.1-2. Holzsarkophage Südrussland: Watzinger 1905, Abb. 65-66, Taf. I. Siehe auch die Bekrönungen von attischen Grabreliefs: Möbius 1929, Taf. 40.2. Ferner die goldene Truhe aus Vergina: siehe oben, Anm. 4. 
Doch auch in diesen Abweichungen von der strengen Symmetrie ergeben sich ornamentale Muster. Die Wendung des Zeus nach seiner rechten Seite wird von Pelops und Hippodameia mit einer Zusammenführung der Kopfwendungen aufgefangen; auf der Gegenseite ergeben die gleichermaßen abgewandten Köpfe von Oinomaos und Sterope ein gedehntes Ausgreifen. Bei dem jungen Paar ist der Mann stärker gebeugt, die Frau stärker aufgerichtet, bei dem Königspaar ist es umgekehrt. Das Gespann des Pelops hat von den Köpfen der Pferde bis zu dem Diener hinter dem Wagen eine klar begrenzte Erstreckung; bei dem Gespann des Oinomaos ist diese Strecke wegen des Fehlens des Lenkers gedehnter, bis zu dem hockenden Seher. Umgekehrt sind die Figuren in der linken Ecke beide stärker nach der Mitte gerichtet, in der rechten Ecke deutlich einander zugewendet.

Offensichtlich sind solche Differenzierungen von $\mathrm{Zu}$ - und Abwendung, Zusammenführung und Dehnung, systolê und diastolê, sehr bewusst als Formen dynamischer Abweichung von der Symmetrie eingeführt. Ganz entsprechende Prinzipien sind von Polyklet in der Gestaltung der Haare umgesetzt worden (Abb. 2.6): Beim Doryphoros weichen die Zangen und Gabeln der Locken über der Stirn ebenfalls von der axialen Symmetrie in einem dynamischen Wechselspiel von Zusammenführung und Dehnung ab. ${ }^{20}$

Dabei ist entscheidend, dass im Ostgiebel von Olympia alle ,ornamentalen Prinzipien zugleich inhaltliche Aussagen sind. Das gilt zum einen für die symmetrische Grundstruktur der Komposition. Sie zeigt, dass mit dem Königspaar und dem Brautpaar zwei ethische ,Welten“ gegeneinander stehen. Ebenso aber gilt es für die dynamischen Abweichungen von der Symmetrie. Die Wendung des Zeus zu Pelops und Hippodameia zeigt an, dass er ihnen Zustimmung und Beistand gewährt. Die gegenseitige Zuwendung der Brautleute führt ihre liebende Zusammengehörigkeit vor Augen. Die Abwendung des Herrscherpaares macht ihre Distanz zu Zeus und ihren Blick auf das kommende Unglück deutlich. Die Seher bringen in ihren Haltungen der $\mathrm{Zu}$ - und Abwendung, des Auf- und Niederblickens die Vision der unterschiedlichen Schicksale zum Ausdruck. Die Flussgötter demonstrieren in den Bewegungen ihrer Körper verschiedene Stadien von Aufmerksamkeit und Erregung. In den formalen Prinzipien der Zusammenführung und Dehnung, systolê und diastolê, kommen ethische und psychische Kräfte zum Ausdruck.

Das wirft auch ein Licht auf die scheinbar rein ornamentalen Gebilde, die hier mit der Komposition des Giebels verglichen worden sind. Auch die vegetabilen Schmuckmotive und die Locken des Doryphoros sind nicht rein formale ,Zier', sondern bringen Kräfte und Prinzipien des ,Lebens' zur Entfaltung. Nikolaus Himmelmann hat gezeigt, dass dem griechischen Ornament seit der Frühzeit ,organische‘ Kräfte eigen sind, und Hans von Steuben hat dargelegt, dass die Lockenformen des Doryphoros dem gesamten kontrapostischen Körperkonzept Polyklets als eines dynamischen Zusam-

20 Steuben 1979, 11-12. 


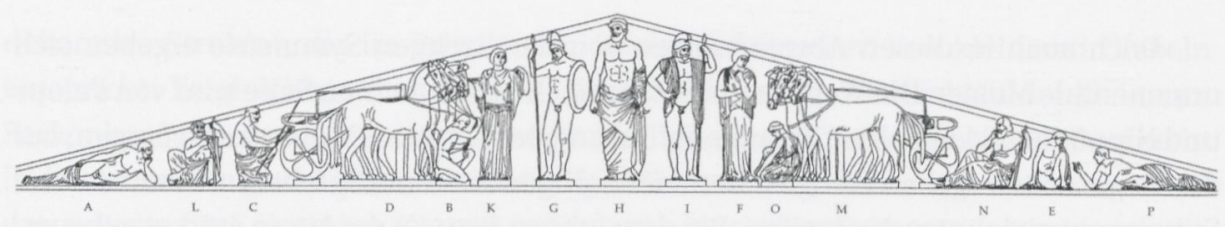

Abb. 2.4: Ostgiebel des Zeus-Tempels von Olympia, Rekonstruktion. Um 460 v. Chr.

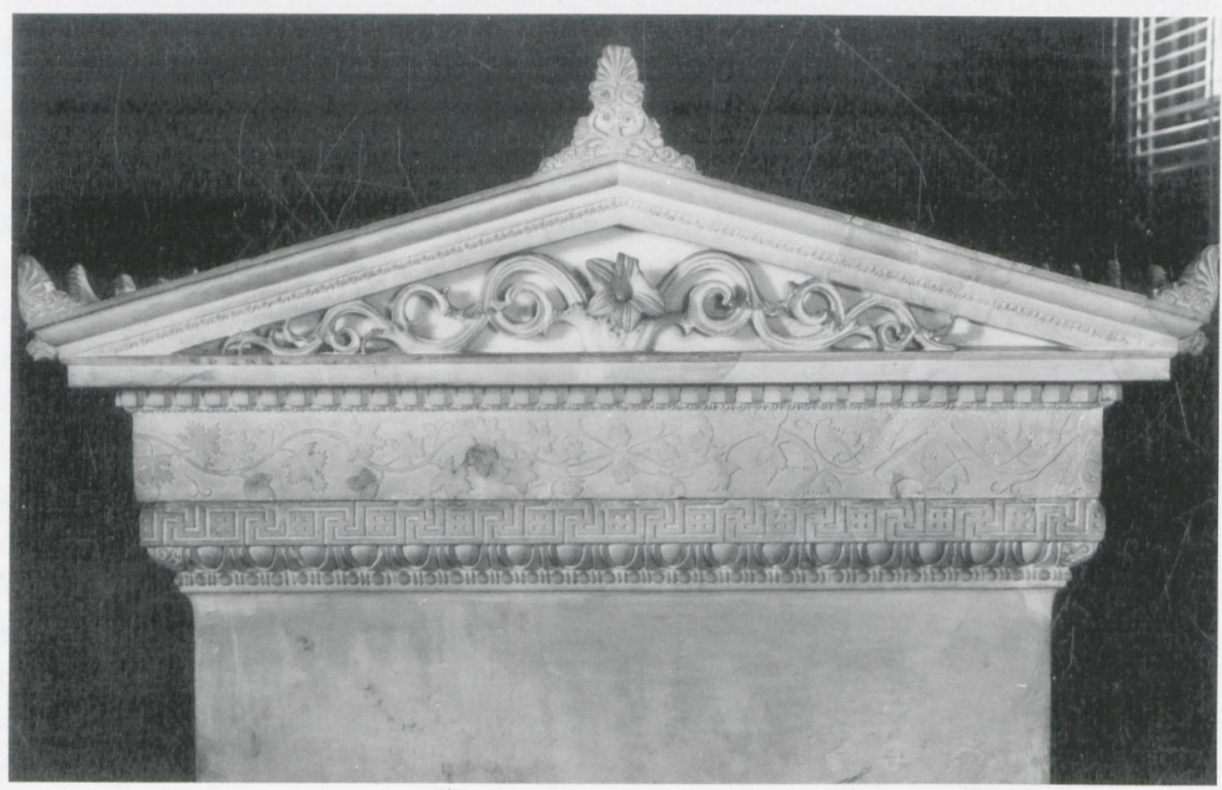

Abb. 2.5: Sarkophag aus dem Grab des Alexander-Sarkophags in Sidon, um 320 v. Chr. Istanbul, Arkeoloji Müzesi.

menspiels von antithetischen Kräften den Anspannung und Entspannung, der Kontraktion und Dehnung entsprechen. Ornamente und Lebewesen gehören derselben Welt konzeptueller Bilder an. ${ }^{21}$

Am Tempel von Olympia ist die Komposition des Giebels nicht nur stark von der Vorgabe des flachen Dreiecks konditioniert. Wie Peter Grunauer gezeigt hat, nimmt sie auch Bezug auf die gesamte architektonische Struktur des Baues, insbesondere auf die Säulen sowie die Triglyphen und Metopen des Gebälks. Die zentrale Figur des Zeus erhebt sich über der mittleren Triglyphe; zu seinen Seiten entsprechen die Männer und Frauen mit den kauernden Dienern den anschließenden Paaren von Metope und Triglyphe. Die beiden Gespanne haben ebenfalls die Ausdehnung einer

21 Himmelmann 1968; Steuben 1979, 11-12. 


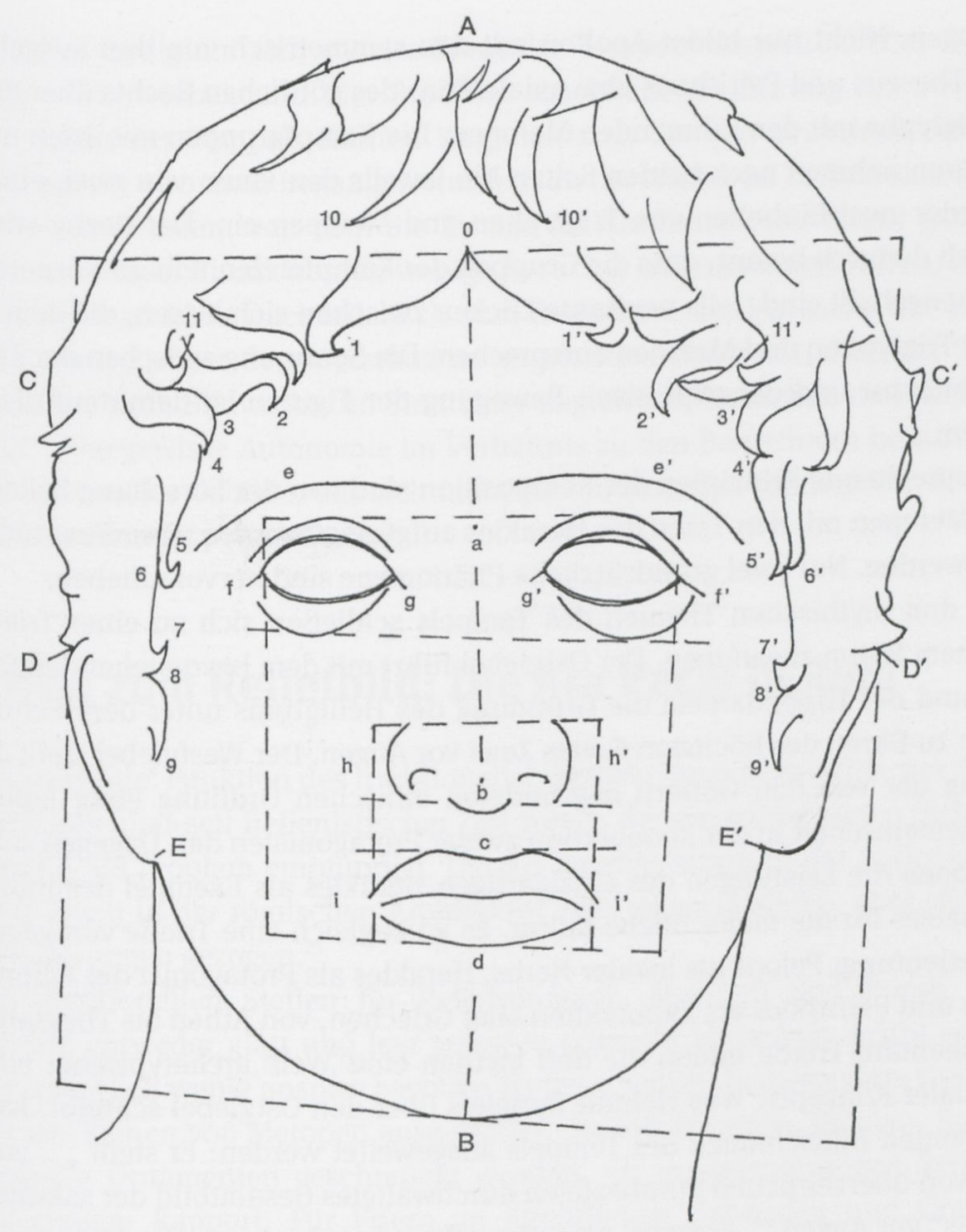

Abb. 2.6: Doryphoros des Polyklet, Schema der Stirnlocken (nach von Steuben 1973), um 440 v. Chr.

Folge von Metope und Triglyphe. Danach nehmen die drei kauernden, sitzenden und liegenden Figuren den Raum über je einer bzw. zweier Metopen oder Triglyphen ein. Die Figuren der Szene im Giebel entfalten ihre Lebendigkeit in einem pointierten Bezug auf die (annähernd) geometrische Serialität der Architektur. ${ }^{22}$

Noch überraschender ist, dass Peter Grunauer entsprechende Prinzipien sogar für den Westgiebel aufgezeigt hat: Auch dort sind die stark bewegten Figuren der Kentauromachie, die sich auf den ersten Blick in ihren wogenden Bewegungen jeder Tektonik zu entziehen scheinen, gleichwohl streng auf den Rhythmus der Architek-

22 Grunauer 1974, 1-51; siehe dazu Ridgway 1999, 80-81. 
tur bezogen. Nicht nur bildet Apollon mit den symmetrisch von ihm ausgehenden Helden Theseus und Peirithoos eine axiale Trias des göttlichen Rechts über der mittleren Triglyphe mit den rahmenden Metopen. Die Kampfgruppen mit ihren markanten Zäsuren nehmen nach beiden Seiten hin jeweils den Raum von zwei, einer, drei und wieder zwei Einheiten von Triglyphen und Metopen ein. Der Bezug wird vielfach noch dadurch betont, dass die Gruppen der Kämpfenden teils zu Körpermassen zusammengeballt sind, teils markante Lücken zwischen sich lassen, die dem Rhythmus der Triglyphen und Metopen entsprechen. Die Spannung zwischen der Tektonik der Architektur und der explosiven Bewegung der Figuren ist damit auf die Spitze getrieben.

Entsprechende Prinzipien der Komposition sind von der Forschung bekanntlich an den Metopen mit den Taten des Herakles aufgezeigt worden. Das muss nicht wiederholt werden. Nur zwei grundsätzliche Phänomene sind hervorzuheben.

Die drei mythischen Themen des Tempels schließen sich zu einer Triade von heroischem Ruhm zusammen. Der Ostgiebel führt mit dem bevorstehenden Sieg des Pelops und der Hippodameia die Gründung des Heiligtums unter dem Schutz und zugleich zu Ehren des höchsten Gottes Zeus vor Augen. Der Westgiebel stellt die Verteidigung der von den Göttern begründeten ethischen Ordnung einer kollektiven Lebensgemeinschaft in der Kooperation zweier Protagonisten dar. Dagegen schildern die Metopen die Leistungen des einzigartigen Herakles als Exempel des individuellen Einsatzes für die menschliche Kultur. Es ist zugleich eine Triade von geographischer Bedeutung: Pelops als lokaler Heros, Herakles als Protagonist der Peloponnes, Theseus und Peirithoos als Exponenten aller Griechen, von Athen bis Thessalien. Als komplementäre Triade bilden die drei Mythen eine ,Welt" archetypischer ethischer und sozialer Konzepte. Was Helmut Kyrieleis über den Ostgiebel schreibt, kann auf den gesamten Bildschmuck des Tempels ausgeweitet werden: Er stellt „... ein komplexes, von überzeitlichen Sinnbezügen durchwaltetes Gesamtbild der sakralen Welt Olympias“ vor Augen. ${ }^{23}$,Kosmos' als Schmuck und als konzeptuelle ,Ordnung“ fallen in eins.

An dem Tempel sind die drei Themen so angebracht, dass die Giebel an Front und Rückseite einander antithetisch gegenüber stehen, während die Metopen eine Klammer zwischen Vorder- und Rückseite darstellen. Dabei ist überraschend wenig dafür gesorgt worden, dass dies Konzept von den Besuchern des Heiligtums visuell wahrgenommen wurde. Nicht nur ist die Sichtbarkeit der figürlichen Bilder durch die allgemeine Anbringung in großer Höhe stark beeinträchtigt, sondern für die Reliefs der Metopen ist nicht einmal der Platz an den Außenfronten, sondern innerhalb der Säulenstellung hoch über den Zugängen zum Pronaos und zum Opisthodom gewählt: wo sie nur aus sehr steilem Winkel und im Schatten der Umgangshalle zu sehen waren. Weiter kam erschwerend hinzu, dass die entscheidenden Rituale und

23 Kyrieleis 2012/2013, 52-84, Zitat S. 79. 
Vorgänge im Heiligtum sich vor der Front des Tempels abspielten: Hier führte der Prozessionsweg vorbei, und hier fand die abschließende Ehrung der Sieger statt. An den Seiten des Tempels führten Wege entlang, die von geweihten Bildwerken gesäumt waren, also auch begangen wurden, an der Rückseite befand sich vor allem der wilde Olivenbaum, von dem die Zweige für die Kränze der Sieger geschnitten wurden. An diesem Ritual werden wenige Besucher teilgenommen haben, und auch sonst wurde man nicht nachdrücklich zum Betrachten der Rückseite des Bauwerks und der Metopen über dem Opisthodom angehalten. Das heißt, dass der Schmuck des Tempels durch Bildwerke, im Sinn eines zugleich ästhetischen und inhaltlichen ,Kosmos', eine gewisse Autonomie im Verhältnis zu den Betrachtern besaß: Der Bau war ein Sinn-volles Gebilde, das sich nicht für die aktuelle, sondern für eine potentielle Wahrnehmung präsentierte. ${ }^{24}$

\section{Der Weg zum Reliefbild: Die Ara Pacis in Rom}

Die ,ornamentale‘ Funktion des traditionellen Reliefschmucks griechischer Architektur wurde offenbar seit hellenistischer Zeit wegen der eingeschränkten Sichtbarkeit zunehmend als Problem empfunden. Daraus wurden schon in der hellenistischen, dann vor allem in der römischen Architektur zwei gegensätzliche, aber sich ergänzende Folgerungen gezogen. ${ }^{25}$

Die traditionellen Stellen für Reliefschmuck, Metopen und Friese, wurden zunehmend entweder glatt und leer gelassen oder mit einfachen, leicht erkennbaren und inhaltlich wenig anspruchsvollen Motiven gefüllt. Insbesondere Grabbauten wurden mit Reihen von Metopen ausgestattet, die mit Rosetten, Phialen, Bukranien und anderen Ornamenten geschmückt wurden, oft in gleichförmigen Serien oder abwechselndem Rapport. Für Friese an öffentlichen Gebäuden und Grabbauten wurden vor allem Serien von Girlanden, daneben variable Reihen von Opferinstrumenten, Waffen, Rüstungsstücken und schließlich vielfältige vegetabile Ranken gewählt. ${ }^{26}$

Eine entgegengesetzte, aber auf dasselbe Problem reagierende Konsequenz führte dahin, Reliefs aus ihrer traditionellen Einbindung in die Architektur zu lösen und sie frei auf Wände zu setzen, wo geeignete Formate für komplexe Bildthemen und eine Platzierung in Sichthöhe der Betrachter möglich waren. Vorbilder dafür konnten in der großen Malerei gefunden werden: Schon im 7. Jahrhundert v. Chr. wurden an den Tempeln von Isthmia und Kalapodi die Außenwände der Cella mit großen Fresko-

\footnotetext{
24 Wege im Heiligtum von Olympia: T. Hölscher 2002, 331-345.

25 Zum Folgenden siehe vorläufig T. Hölscher 2009, 63-66. Das Phänomen bedarf weiterer Untersuchungen.

26 Honroth 1971; Hesberg 1981, 201-245; Schörner 1995; Polito 1998; Grüßinger 2001; Maschek 2012.
} 
Malereien geschmückt; daneben gab es in geschützten Innenräumen großformatige Tafelgemälde, die im 5. Jahrhundert v. Chr. im Kreis von Polygnot und Mikon zu einer ersten Blüte geführt wurden. Entscheidend ist, dass dieser gemalte Bildschmuck nicht in tektonisch vorbestimmte ,Schmuck-Zonen“ eingefügt, sondern in freier Verfügung auf den Wänden platziert wurde. ${ }^{27}$

In diesem Sinn wurden dann seit dem frühen Hellenismus zunehmend wandartige Sockel und Wände mit Reliefs ausgestattet. Marksteine dieser Entwicklung sind das Neorion von Delos, aus dem frühen 3. Jahrhundert v. Chr., mit einem langen monumentalen Fries von Tritonen und Nereiden am Sockel des geweihten Kriegsschiffes von Demetrios Poliorketes; dann der große Altar von Pergamon, mit dem Gigantenfries außen am Sockel unter den Hallen und dem Telephos-Fries im Inneren in den Hallen des Hofes umlaufend; ebenfalls in Pergamon das Propylon zum Heiligtum der Athena, wo die Balustraden im Obergeschoss mit Reliefzenen geschmückt waren, darunter einer figurenreichen Darstellung des Baues des Troianischen Pferdes. Schließlich wurden in Rom seit dem späteren 2. Jahrhundert v. Chr. Grabbauten mit großen Relieftafeln an den Außenwänden geschmückt: teils mit einfachen emblematischen Motiven, wie das Grab des Konsuls Sergius Sulpicius Galba mit den 12 fasces seines Konsulats, teils mit komplexen Kompositionen, wie die Grabexedra eines Feldherrn, wahrscheinlich Publius Ventidius Bassus, mit der Darstellung einer Schlacht und verschiedenen allegorischen Figuren. Gleichzeitig wurde seit dem späteren 2. Jahrhundert die Gattung der klassizistischen Schmuckreliefs entwickelt, die zum Dekor vornehmer Wohnsitze in die Wände von repräsentativen Räumen eingelassen wurden. Aus solchen Wurzeln entstanden verschiedene Formen des ,Reliefbildes‘, das als anspruchsvolle Art des Dekors in der öffentlichen und privaten Architektur des Römischen Reiches eingesetzt wurde. ${ }^{28}$

Das erste erhaltene Denkmal, das diese Möglichkeiten des Reliefschmuckes voll nutzt, ist die Ara Pacis (Abb. 2.7). Das komplexe Geflecht von ornamentalen und figurativen Motiven dieses Bauwerks ist oft genug analysiert worden: Hier sollen nur wenige Phänomene benannt werden, die einerseits die große neue Freiheit ,repräsentativer' Reliefbilder betreffen, andererseits gleichwohl deren heteronome Prägung durch ihre ,decorative“ Einbindung hervorheben. ${ }^{29}$

Die Umfassungswand ist in einer bisher nicht bekannten Weise mit großformatigen Reliefbildern bedeckt. Die Reliefplatten zu Seiten der Eingänge an der Vorderund Rückseite bieten einen Bildraum von völlig neuen Dimensionen für die komple-

27 Zu griechischen Wand- und Tafelgemälden siehe Moreno 1987; Rouveret 1989; Scheibler 1994, bes. $14-35$.

28 Neorion, Delos: Webb 1996, 134-136. Grabbau des Ser. Sulpicius Galba: Schäfer 1989, 363, A 1. Grabexedra des P. Ventidius Bassus: Sydow 1974. Schmuckreliefs: Froning 1981. Zu Reliefbildern siehe auch in diesem Band den Beitrag von Reinhardt.

29 Zur Ara Pacis siehe immer noch: Simon 1967 und La Rocca 1983. Neuerdings: Rossini 2006; Mlasowski 2010; Simon 2010. 


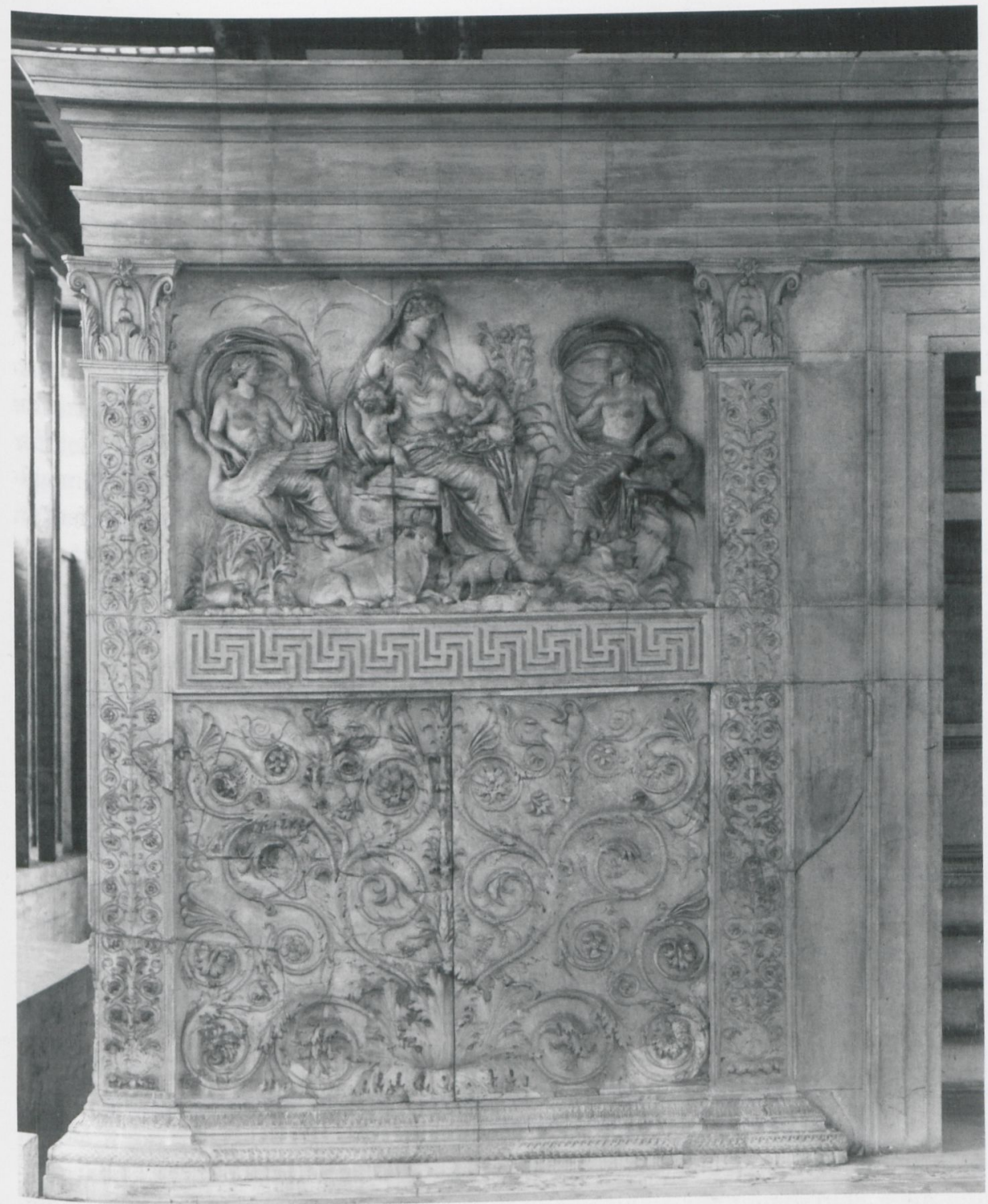

Abb. 2.7: Ara Pacis Rom, Ostseite, linke Hälfte, 13-9 v. Chr.

xen Szenen der Mythen von Aeneas und den Zwillingen und der Allegorien von Tellus und Roma. Selbst die großen Friese der Langseiten mit der zweifachen Prozession der staatlichen Würdenträger und der kaiserlichen Familie sind über das Format der traditionellen Friesbänder hinaus zu monumentalen Reliefbildern gesteigert.

Gleichwohl entfalten sich die Szenen nicht als ,freie' Konstellationen von Figuren und Handlungen. Die Bilder der Rückseite sind jeweils als stark hierarchische Kompositionen angelegt, mit einer Hauptfigur und zwei rahmenden Seitenfiguren in 
dynamisch variierender Responsion: auf der linken Seite Italia, nach rechts sitzend, mit den komplementären Aurae des Süß- und des Meereswassers, die die Szene links zur Ecke hin abschließen, rechts zum Eingang hin stärker öffnen; auf der Gegenseite Roma, zu ihren Seiten die Genien des Senats und des Volkes. Die komplementäre Symmetrie beider Reliefs wird in der antithetischen Richtung des Sitzens der beiden Hauptfiguren deutlich: ein ,ornamentales“ Prinzip, das in der sehr summarischen Wiedergabe des Baues auf Sesterzen Neros besonders hervorgehoben ist. ${ }^{30}$

Die dynamische variierende Symmetrie der Figurenszene wird in den Relieffeldern darunter von reichen Rankenkompositionen getragen, die eine sehr viel strengere Symmetrie entfalten. Am klarsten sind die Responsionen auf der Seite des TellusReliefs zu erkennen: Aus einem Akanthuskelch, der in seiner Ausladung etwa der sitzenden Göttin darüber entspricht, steigt ein senkrechter Stängel auf, der auch für die Szene darüber die ideale Mittelachse angibt; daneben entfalten sich die vegetabilen Voluten nach den Seiten, gipfelnd in zwei Schwänen und zwei rahmenden Palmetten, die den Zäsuren der figürlichen Komposition darüber entsprechen. Die strenge axiale Symmetrie des Rankenfeldes und die dynamisch variierende Symmetrie der Figurenszene stehen zueinander in einem komplementären Verhältnis der ornamentalen Gliederung. ${ }^{31}$

Die Prozession auf dem Südfries gibt bekanntlich die Supplicatio zur Rückkehr des Augustus aus Gallien und Spanien 13 v. Chr. in einer idealen Besetzung wieder, unter Einschluss von Drusus, der zu dieser Zeit in Germanien Krieg führte. Sie ist so gegliedert, dass die beiden Hauptfiguren, Augustus und Agrippa, jeweils im gleichen Abstand von der Mittelachse, drei konstitutive Abschnitte markieren: Vor dem Kaiser dominieren die Lictoren als Personal seiner politischen Machtstellung, in der Mitte treten die Flamines als oberste religiöse Würdenträger auf, hinter Agrippa folgt die kaiserliche Familie. In der Antike müssen die drei Gruppen zusätzlich durch die ursprüngliche Bemalung der Gewänder gegeneinander abgesetzt gewesen sein. An den Scharnierstellen steht Augustus als Referenzfigur für die Lictoren im ersten Abschnitt und als Beginn der folgenden Priesterschaft, Agrippa als Endpunkt der Priester und als Beginn der kaiserlichen Familie. Weiterhin sind die beiden Protagonisten dadurch herausgehoben, dass sie von Personen in drei - statt der üblichen zwei - Reliefschichten umgeben werden.

Auch hier kommt die Ambivalenz von Repräsentation und Dekor zur Geltung. Einerseits ist die gesamte Ausrichtung des Zuges nach links ,autonom‘ vom Thema diktiert, die Reihenfolge der teilnehmenden Gruppen ist an der Realität des Rituals orientiert, die $\mathrm{Zu}$ - und Abwendung der Teilnehmer verleiht dem Ritual lebendige

30 Sesterze Neros: Simon 2010, Abb. 6; La Rocca 1983, Abb. S. 12.

31 Die Rankenfelder der Ara Pacis sind in neuerer Zeit Gegenstand weitreichender Ausdeutungen mit Referenz auf die figürlichen Friese darüber geworden: Castriota 1995; Sauron 2000; Sauron 2013. Darauf kann hier nicht näher eingegangen werden. 
Variation. Auch die Verdichtung der umgebenden Figuren bei Augustus und Agrippa entspricht insofern der realen Praxis, als der Kaiser und andere politische Protagonisten bei öffentlichen Auftritten durch dichtes Geleit in ihrer Bedeutung hervorgehoben wurden. Andererseits wird die visuelle Gliederung heteronom von den Vorgaben des Baues bestimmt: Augustus und Agrippa korrespondieren einander, symmetrisch zu der imaginären Mittelachse des Frieses, als Scharniere zwischen den drei Gruppen der Lictoren, der Priester und der kaiserlichen Familie, die in einem Verhältnis von $4: 3: 4$ stehen. Und auch hier steht die dynamisch variierte Symmetrie der figürlichen Szene in komplementärer Spannung zu dem vegetabilen Dekor im Feld darunter: Das zentrale Motiv von Akanthuskelch und vertikalem Stängel realisiert die Mittelachse, die im Figurenfries eher imaginär impliziert ist, während die folgenden Nebenachsen zu beiden Seiten, wieder mit bekrönenden Schwänen und Palmetten, einen regelmäßigen Rhythmus vorgeben, der den aufrecht schreitenden Figuren der Prozession eine ornamentale Struktur unterlegt. ${ }^{32}$

Analoge Phänomene sind an den übrigen Elementen des figürlichen und ornamentalen Schmuckes der Ara Pacis zu erkennen. Auch an diesem Denkmal aber schließt sich der Dekor, wie in zahlreichen eingehenden Interpretationen hervorgehoben wurde, zu einer ,Welt' des augusteischen Friedens, in dem sich die mythische Vorzeit mit dem Opfer des Aeneas und der Geburt von Romulus und Remus, die ideale Gegenwart mit dem Kaiser, den Vertretern der römischen Oberschicht in den großen Priesterschaften und der kaiserlichen Familie, und schließlich die Allegorien von Hauptstadt und Reich zu einem umfassenden Panorama vereinigen. Wieder ist dies Kosmos im Sinn von Wert und Ordnung.

Auch die neue Form des großformatigen Reliefbildes ist jedoch nicht durchweg zur Erreichung von nahsichtiger Erkennbarkeit eingesetzt worden. Die Traians- und die Marcus-Säule mit ihren narrativen Reliefbändern entziehen sich durch ihre Höhe von $100 \mathrm{Fuß}$ und den extrem steilen Sichtwinkel weitgehend der genauen Betrachtung. Die Forschung hat aus dieser Tatsache bekanntlich extrem unterschiedliche Folgerungen gezogen: Paul Veyne hat die eingeschränkte Sichtbarkeit gegen die komplexen Interpretationen der Archäologen ins Feld geführt; dagegen hat Martin Galinier die komplexen Bedeutungen der Reliefs zum Anlass genommen, um entsprechend gute Sichtbedingungen zu rekonstruieren. Ein angemessenes Verständnis ist aber wohl nur zu erreichen, wenn man beide Realitäten anerkennt: komplexe Bedeutung und eingeschränkte Sichtbarkeit. Das scheinbare Paradox dieses Befundes kann grundsätzlich analog zum Parthenon - ebenso wie zu gotischen Kathedralen, dem Monument für Vittorio Emmanuele II. in Rom oder dem Mausoleum von Mao Zedong - mit dem oben dargelegten Konzept des Dekors verständlich gemacht werden: Die Bilder konstituieren den ,Sinn' des Denkmals als vollständig ausgearbei-

32 Kaiser und dichtes Geleit: Millar 1977, 110-122. 
tetes Konzept, ihre Wahrnehmung wird in einer Verbindung von partieller Sicht und komplettierender Überzeugung vollzogen. ${ }^{33}$

\section{Standbilder und Architektur: Der ,Kaisersaal‘ von Side}

Standbilder hatten in Griechenland seit dem Beginn der Skulptur in großem Format feste Orte, an denen sie ihre Aufgabe der Repräsentation erfüllten: vor allem Heiligtümer, Gräber, später auch die Agora und öffentliche Anlagen von verschiedener Funktion. Besonders in den Heiligtümern traten sie seit der Frühzeit in Beziehung zu den funktionalen Anlagen, im Lauf der Zeit insbesondere zur sakralen Architektur, später auch zu verschiedenen öffentlichen Gebäuden. Seit dem Hellenismus und insbesondere in der römischen Kaiserzeit führte dieser Prozess bekanntlich zu einer dichten Integration von Standbildern und anderen Bildwerken in die öffentliche Architektur, in deutlicher Korrespondenz zu der geschilderten Entstehung des Reliefbildes. Der übergreifende Begriff dafür ist ,ornamentum urbis‘. Damit kommt die Spannung zwischen autonomer Repräsentation der Bilder und heteronomem Dekor der Architektur besonders pointiert zur Geltung. ${ }^{34}$

Die damit bezeichnete Entwicklung bedarf einer systematischen Untersuchung, die hier nicht geleistet werden kann. Im Folgenden werden nur kurz einige wesentliche Schritte benannt.

Seit dem Wiederbeginn der griechischen Bildkunst im 9. und 8. Jahrhundert v. Chr. wurden kleinformatige Bildwerke in spezifischen Kontexten verwendet, vor allem als Beigaben in Gräbern und Votive in Heiligtümern. Dort bildeten sie kohärente Ensembles, die in ihren Themen und Formen auf die kulturellen Orte der Gräber und Heiligtümer bezogen waren. Mit dem Aufkommen der Großplastik traten dann die Standbilder an Gräbern, in Heiligtümern und zunehmend in anderen öffentlichen Bereichen wie der Agora durch ortsfeste Aufstellung in einen dauerhaft fixierten visuellen Bezug: Es entstanden Räume, in denen lebende Menschen und Bildwerke in religiösen, sepulkralen, politischen und sozialen Praktiken zueinander in Interaktion treten konnten. In den Heiligtümern der archaischen Zeit stellten die Standbilder Gottheiten als Adressaten der kultischen Handlungen, ideale Mitglieder der Gesellschaft als beobachtende Teilnehmer der Rituale, wilde Tiere und Monster als beherrschte Gegenbilder der religiösen und kulturellen Lebensordnung vor Augen. Als bildliche Partner und Zeugen der rituellen Vorgänge wurden sie vor allem in den Zonen aufgestellt, wo die Rituale stattfanden; am Eingang zum Heiligtum, wo sie die

33 Veyne 1988; Veyne 1990; Settis 1992, 40-52; Galinier 2007; demnächst T. Hölscher 2018, Kapitel 6.

34 Ornamentum urbis: Bravi 2014. 
Besucher ,empfingen', und entlang des Hauptweges zum Tempel, wo sie die Prozessionen als ,Betrachter‘ säumten, wie etwa im Heraion von Samos oder im Zeus-Heiligtum von Olympia; und um den Raum des Altars, wo sie zusammen mit den lebenden Teilnehmern das Opfer ,umgaben', wie etwa im Asklepios-Heiligtum von Epidauros. Eine ähnliche ,konzeptuelle Präsenz' wurde verdienten Persönlichkeiten in Form von Ehrenstatuen auf der Agora und den Toten in Form von Grabstatuen in den Nekropolen vor den Toren der Städte gegeben. Diese Standbilder dienten in hohem Maß einer autonomen Repräsentation, in Responsion zu den lebenden Mitgliedern der Gesellschaft - allerdings immer in klarem inhaltlichem Bezug zu den Anlagen und Architekturen der betreffenden sakralen, politischen und sozialen Räume. ${ }^{35}$

Seit (spät-)klassischer und hellenistischer Zeit führte eine dominierende Tendenz dahin, den Charakter der öffentlichen Räume durch neue Formen der Inszenierung visuell zur Wirkung zu bringen. Die Bauwerke der Heiligtümer wie der Agorai wurden absichtsvoll im Hinblick auf eindrucksvolle Fassaden gestaltet, die Bildwerke wurden ihnen als bildliche ,Bewohner' zugeordnet. Zunehmend wurde die Platzierung von Bildwerken in den architektonisch gestalteten Räumen auf visuelle Wirkung hin ausgerichtet: Vor allem in hellenistischen Städten wurde der Ort mit der größten Sichtbarkeit, ,epiphanestatos topos', zum entscheidenden Kriterium für die Aufstellung von öffentlichen Ehrenstatuen und anderen Bildwerken. Das stärkste neue Element waren lange Hallen mit Säulenstellungen, die als Kulissen für sukzessive Aufstellung von Standbildern genutzt wurden. Ein charakteristisches Beispiel ist die Stoa an der Ostseite der Zugangsstraße zum Apollon-Heiligtum von Delos, die im 3. Jahrhundert v. Chr. an den Ecken von zwei markanten pergamenischen Siegesmonumenten eingefasst wurde, zwischen denen dann der Raum mehr und mehr mit Ehrenstatuen gefüllt wurde. Zwar ohne einheitliche Planung, aber aufgrund eines allgemeinen Grundkonzepts entstand auf dieser Weise eine Art von sich selbst generierender visueller Gesamtwirkung. Eine ähnliche autopoietische Integration von Architektur und Standbildern findet sich etwa an der Agora von Priene, die Übernahme der Praxis im späthellenistischen Italien ist an der Südseite des Forums von Pompeii zu beobachten. Wie intentional dies Zusammenspiel von Bau- und Bildwerken nicht nur gestaltet, sondern auch wahrgenommen wurde, wird aus den bekannten Fresken aus den praedia der Iulia Felix in Pompeii deutlich, auf denen die rahmenden Kolonnaden um das Forum der Stadt mit den Reiterstatuen vor den Intercolumnien zu eindrucksvoller Wirkung gebracht sind. Agorai, Heiligtümer und andere öffentliche Anlagen entwickelten sich zu architektonischen Bühnen, auf denen die Bildwerke, obgleich diachron und in einzelnen Initiativen aufgestellt, zu synchronen Bild-Gemeinschaf-

35 Einiges dazu bei T. Hölscher 2015a. Zu Standbildern in archaischen Heiligtümern siehe Duplouy 2006, 185-216; Franssen 2011; T. Hölscher 2015b; Bildwerke und rituelle Räume im Heiligtum von Olympia: T. Hölscher 2002. 
ten von ruhmreichen Menschen, zum Teil zusammen mit Göttern und Heroen, zusammenwuchsen. ${ }^{36}$

In der Konsequenz dieser Entwicklung begann man dann, architektonische Komplexe schon von Anbeginn mit einheitlichen ,Programmen‘ von Standbildern und anderen Bildwerken auszustatten. Ein frühes Beispiel ist in Rom das Grab der Scipionen, das um die Mitte des 2. Jahrhunderts v. Chr. mit einer neuen Fassade versehen wurde: Darin waren, vielleicht in drei Nischen, Bildnisstatuen des P. Cornelius Scipio Africanus maior, des L. Cornelius Scipio Asiaticus und angeblich ihres Ruhmesdichters Ennius aufgestellt. ${ }^{37}$ Besondere Bedeutung für diese Praxis hatten die temporären Theaterbauten für die aufwändigen Spiele aus Anlass von Triumphen seit dem 2. Jahrhundert v. Chr., in deren Szenenfronten die erbeuteten Bildwerke vor allem aus Griechenland ausgestellt wurden: Den oft zitierten Höhepunkt stellte der Bau des M. Aemilius Scaurus von 58 v. Chr. dar, mit einer Fassadenarchitektur von 360 Säulen und insgesamt 3000 Standbildern aus Bronze. In denselben Jahren setzte Pompeius, nach seinem Triumph über Mithridates $61 \mathrm{v}$. Chr., in seiner monumentalen Theateranlage mit der anschließenden Gartenporticus auf dem Marsfeld ein derartiges Konzept in eine dauerhafte Architektur mit einem komplexen Programm von Bildwerken um. Aus schriftlichen Quellen ist bekannt, dass drei Tempel mit Kultbildern für Venus Victrix, Virtus und Felicitas die Cavea bekrönten und die ganze Anlage unter das Motto von Sieg und Glück stellten. Vierzehn monumentale Standbilder repräsentierten die nationes, die Pompeius in den Kriegen gegen Mithridates unterworfen hatte; eine andere Gruppe von kolossalen Statuen stellte die neun Musen dar, die in der erkämpften Friedenszeit das Leben bestimmen sollten. Bilder von historischen Dichterinnen, dazu von berühmten Hetären und von mythischen Müttern, die ungewöhnliche Ausgeburten zur Welt gebracht hatten, evozierten eine Atmosphäre der Venus in allen ihren Facetten. Hinzu kamen vier originale griechische Gemälde, die Kadmos und Europa, Alexander den Großen, einen angreifenden Krieger und ein Opfer von Stieren als Exempel der Weltherrschaft, der militärischen virtus und der pietas darstellten. Die Quellen lassen nicht genau erkennen, wo die verschiedenen Bildwerke innerhalb der Anlage aufgestellt waren. Deutlich ist aber, dass sie alle ihren Referenzpunkt in einer Bildnisstatue des Pompeius selbst hatten, die in einem Saal in der Achse der Porticus stand, der als Versammlungsraum des Senats dienen sollte. ${ }^{38}$

Die Theateranlage des Pompeius mit ihren vielen Bildwerken, teils älteren griechischen Gemälden teils neu gefertigten Standbildern, wurde zuletzt wieder als

36 Sichtbarkeit und epiphanestatos topos: Bielfeldt 2012. Hellenistische Städte: Ma 2013. Delos, spätklassisch und hellenistisch: Griesbach 2013. Priene: Raeck 1995; Bielfeldt 2012. Fresken in der praedia der Iulia Felix in Pompeii: Olivito 2013.

37 Coarelli 1988; Giuliani 1986, 163-189; Papini 2004, 395-400.

38 Zu den Bildwerken im Pompeius-Theater siehe Coarelli 1971-1972; Fuchs 1981-1982; Sauron 1987; Bravi 2014, 73-84 (mit weiterer Lit.). 
„Pompey’s Museum“ für „Culture and History“ betitelt und gedeutet. Entsprechend wird die verbreitete Praxis, Meisterwerke der griechischen Malerei und Skulptur in öffentlichen Gebäuden Roms auszustellen, immer wieder nach dem Konzept einer „musealen“ Ausstellung von „Kunst“ für das gebildete Publikum der Stadt Rom erklärt. Dem ist entgegenzuhalten, dass das Konzept des „Museums“ als eines ausgegrenzten Raumes von „Kunst“ und „Bildung“ ein neuzeitliches Phänomen ist, und dass in der Antike alle Bildwerke in einem fundamentalen Sinn als Faktoren des religiösen, sozialen und politischen Lebens in die öffentlichen und privaten Räume der Gesellschaft eingebunden waren. Die Theateranlage des Pompeius zeigt in exemplarischer Weise, dass die Bildwerke dazu dienten, diesen öffentlichen Raum als eine ,Welt' der römischen Herrschaft in Sieg und Glück zu definieren. ${ }^{39}$

Derartige konzeptuelle Bilder-Welten sind mit Hilfe schriftlicher Zeugnisse in zahlreichen anderen Bauwerken der römischen Kaiserzeit zu rekonstruieren:

Der Tempel der Concordia am Forum Romanum wurde von seinem Bauherrn Tiberius mit einer höchst bewusst gewählten Auswahl von Götterstatuen griechischer Bildhauer ausgestattet, die die Nischen in der Cella schmückten und die Treppen der Front flankierten: Zeus, Hera und Athena als Äquivalente zur Kapitolonischen Trias; Apollon und Leto mit den Zwillingen als Götter des Palatin, dazu Apollons Sohn Asklepios; Demeter, Ares und Hestia als Äquivalente zu den Gottheiten der Regia, Mars und Ops Consiva, sowie zu dem römischen Ur-Heiligtum der Vesta, am Ostrand des Forum. ${ }^{40}$

In entsprechender Weise beschreibt Pausanias den Innenraum des Heraion von Olympia, wo die Nischen zwischen den Innensäulen zu seiner Zeit mit alten Standbildern geschmückt waren, die aus ihren ursprünglichen Kontexten herausgelöst worden waren. In ihrer neuen Konstellation stellten sie eine sehr bewusst konzipierte Bilderwelt der Kulte von Mädchen und Frauen vor Augen, die sich in Olympia um die Göttin Hera formierten. Auch hier ist ,Decor‘ nicht rein ästhetische Zier, sondern sinnstiftender Schmuck. ${ }^{41}$

Der Schritt von sukzessiv gewachsenen Ensembles zu homogen konzipierten ,Programmen' ist anschaulich in Rom zu beobachten: Dort waren am republikanischen Forum die Ehrenstatuen und andere Bildwerke seit dem 4. Jahrhundert v. Chr. zu einem exemplarischen Panorama historischer, mythischer und göttlicher Figuren der res publica akkumuliert worden; daraus wurde dann am Forum des Augustus ein systematisches Konzept einer ,vollständigen‘ Präsenz großer Männer Roms ent-

39 Gegen das Konzept antiker ,Museen` siehe T. Hölscher 1989; T. Hölscher 1994; T. Hölscher 2015a, 205-214; Bravi 1998.

40 Zur Bild-Ausstattung des Concordia-Tempels siehe Kellum 1990; Bravi 1998; Celani 1998, 125-132 und 209-213 (Deutung im Sinn eines Museums); Bravi 2014, 185-201; demnächst T. Hölscher 2018, Kapitel 6.

41 Paus. 5.17.1-4. Dazu Krumeich 2008; Hupfloher 2012; demnächst T. Hölscher 2017. 


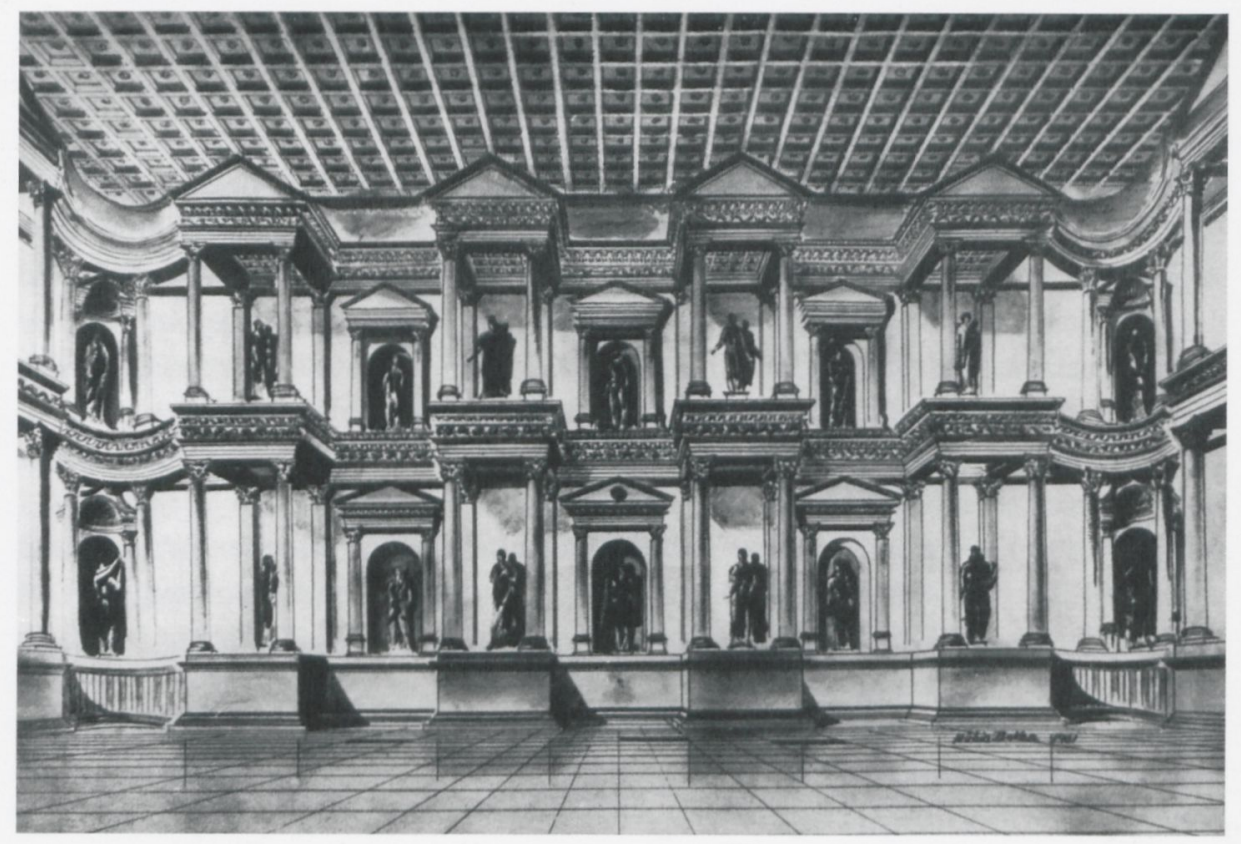

Abb. 2.8a: Sog. Kaisersaal von Side, Mitte 2. Jh. n. Chr.

\begin{tabular}{|c|c|c|c|c|c|c|c|c|c|c|c|c|}
\hline$?$ & $?$ & Kore & $\begin{array}{c}\text { Sand.- } \\
\text { Binder }\end{array}$ & $\begin{array}{c}\text { Disk. } \\
\text { Ludovisi }\end{array}$ & (Apox.?) & Hermes & Diadum. & $\begin{array}{c}\text { Disk. } \\
\text { Myron }\end{array}$ & $?$ & $?$ & Apollo & Marsyas \\
\hline $\begin{array}{c}\text { KI. } \\
\text { Herc. }\end{array}$ & Nike & Demeter & $?$ & $\begin{array}{c}\text { Hermes } \\
\text { Kyr.-Per. }\end{array}$ & $\begin{array}{c}\text { Port. } \\
\text { Stat. }\end{array}$ & $\begin{array}{c}\text { Port. } \\
\text { Stat. }\end{array}$ & $\begin{array}{c}\text { Port. } \\
\text { Stat. }\end{array}$ & $\begin{array}{c}\text { (Ares } \\
\text { Borgh.?) }\end{array}$ & Doryph.? & Nemesis & Hygieia & Asklep. \\
\hline
\end{tabular}

Abb. 2.8b: Verteilung der gefundenen Skulpturen (Rekonstruktion T. Hölscher).

wickelt: von den Anfängen bis zur Gegenwart, in chronologischer Sequenz, in der Antithese von berühmten Familien und gens Iulia, und in konsequentem Bezug zu der Architektur des Raumes. Aus dem gewachsenen politischen Gedächtnis der Republik wird ein geschlossenes geschichtliches Konzept. ${ }^{42}$

Ähnliche Phänomene sind von der Forschung seit längerer Zeit für verschiedene Bautypen der römischen Architektur aufgezeigt worden, vor allem für Theater und Thermen. ${ }^{43}$ Ein konkretes Beispiel, das zugleich die weite Verbreitung der Praxis im Römischen Reich bezeugt, ist der so genannte Kaisersaal an der Staatsagora von Side (Abb. 2.8a-b). Der monumentale Versammlungsraum, der unter anderem dem Kaiserkult diente, war im Inneren mit einer prachtvollen Blendarchitektur in zwei

42 Standbilder Forum Romanum: Sehlmeyer 1999; Walter 2004, 139-143. Augustus-Forum: Zanker 1968; Spannagel 1999.

43 Fuchs 1987; Manderscheid 1981. 
Stockwerken geschmückt, in deren Nischen ein vielgestaltiges Ensemble von Porträtstatuen und Idealfiguren, vor allem Kopien nach griechischen Originalen, aufgestellt war. Wenngleich die Bildwerke nicht vollständig gefunden wurden und die Fundorte nicht bei allen genau bekannt sind, so lässt sich doch aus den einigermaßen gesicherten Daten eine ungefähre Vorstellung von dem Konzept gewinnen. ${ }^{44}$

Im Zentrum, wohl in der unteren Etage, standen die Porträtfiguren. Die Köpfe sind verloren, doch muss es sich wohl um Darstellungen des regierenden Kaisers, wahrscheinlich Antoninus Pius, zusammen mit seinen vorgesehenen Nachfolgern gehandelt haben. In ihrer unmittelbaren Umgebung waren sie gerahmt von Figuren der gymnasialen Ausbildung der Jugend: den antithetischen Diskuswerfern des Myron und der Sammlung Ludovisi, dem Apoxyomenos des Lysipp, sowie dem Hermes Kyrene-Perinth und dem Diadoumenos des Polyklet. In den Nischen der Ecken standen, als korrespondierende Mächte, links Demeter und Kore als Gottheiten der agrarischen Fruchtbarkeit, rechts Nemesis, wohl als Gottheit der kriegerischen Rache gegen äußere Feinde. An der linken Seitenwand ist aus den Figuren der ,Kleinen Herculanerin' und einer Nike kein klares Konzept zu erkennen, an der gegenüberliegenden Wand dagegen ergibt sich mit Apollo und Marsyas sowie Apolls Sohn Asklepios eine Gruppe, die die Vorstellungen von göttlichem Recht und physischem Heil umfasst. Die weiteren, nicht genauer lokalisierten Figuren aus dem Saal, Ares Borghese, Hera vom Typus Ephesos, Hygieia sowie Herakles (Keule), lassen sich ohne Probleme in dies Programm einfügen.

Auch der Concordia-Tempel in Rom und das Heraion in Olympia mit ihren griechischen Bildwerken wurden immer wieder als antike Museen gedeutet, und sogar die Skulpturen des Kaisersaales von Side wurden als eine kunstgeschichtlich orientierte Auswahl von Kopien nach Meisterwerken der größten griechischen Bildhauer gewertet. Dagegen sprechen sehr deutlich die inhaltlichen Konzepte, die in der Auswahl und Anordnung der Werke deutlich werden: Überall ist sinnvolle Selektion der Bildthemen zu erkennen, nirgends kommt es zu zufälligen oder redundanten Themen, die durch rein ästhetische Kriterien verursacht wären, und wo die Aufstellungen rekonstruierbar sind, sind inhaltliche Konstellationen zu erkennen.

Das heißt nicht, dass solche Konzepte von den Betrachtern immer als solche wahrgenommen wurden. Damit stellt sich die allgemeine Frage nach der spezifischen Wirkung von Dekor und Ornament.

44 Die Skulpturen bei Inan 1975, Nr. 1, 2, 3, 4, 7, 10, 12, 20, 22, 28, 32, 36, 37, 54, 55, 67, 69, 77, 94, 345, 421, 422, 423, 424 und 425. Dazu Rezension von Linfert 1979; Linfert 1995. 


\section{Schluss: Dekor, Wert, Sichtbarkeit}

Für den modernen Betrachter ist der figürliche Schmuck der griechischen und teilweise auch der römischen Architektur durch eine eklatante Diskrepanz zwischen künstlerischem Rang und thematischer Komplexität auf der einen Seite und eingeschränkter Sichtbarkeit auf der anderen Seite geprägt. ${ }^{45}$

Die Einschränkung der Sichtbarkeit wird durch mehrere Faktoren hervorgerufen: Generell sind Metopen und Friese, Giebel und Akrotere, wie bereits hervorgehoben, am Bau in großer Höhe, das heißt für Sicht aus mehr oder minder steilem Winkel angebracht. Zudem sind am Zeus-Tempel von Olympia die Metopen, am Parthenon und am Hephaisteion in Athen die Friese nur entweder von außen durch die Intercolumnien der Säulen oder aus dem Inneren der Peristase in extrem ungünstiger Höhe zu sehen. Zahlreiche Bauten, wie die Schatzhäuser von Siphnos und Athen in Delphi, waren nicht von allen Seiten zu umgehen, so dass die Friese bzw. Metopen einer ganzen Längsseite überhaupt nicht zu sehen waren. In anderen Heiligtümern, etwa der Akropolis von Athen, waren der Zugang, der Prozessionsweg und der Altarplatz so gelegen, dass in der normalen Situation der Wahrnehmung des Parthenon die beiden Fassaden und die nördliche Langseite, nicht aber die südliche Seite in den Blick kamen; wie viele Besucher zusätzlich die Südseite umschritten, ist zumindest fraglich. Dasselbe gilt für das Erechtheion mit seinem vielfach abgewinkelten Fries, der nur durch Herumgehen auf verschiedenen Gehniveaus, mit Betreten und Verlassen verschiedener angrenzender sakraler Bezirke, ganz zu betrachten war. Am Parthenon kommt hinzu, dass das ,Programm‘ des Bildschmucks nur bei mehrmaligem Umschreiten zu verstehen war; insbesondere der Fries mit der doppelten Prozession von der Südwest-Ecke um beide Seiten der Cella bis zur Mitte der Ost-Seite erforderte eine zweifache Umgehung. Wenn man berücksichtigt, dass die normalen Besucher der Akropolis nicht zum Studium von Bildprogrammen, sondern zur Teilnahme am Kult kamen, dann wird man bei den meisten Betrachtern kaum übermäßige Anstrengungen voraussetzen, um die Schwierigkeiten des Sehens zu überwinden.

In anderer Weise war die Wahrnehmung der bildlichen Ausstattung in römischen Anlagen und Architekturen wie den Kaiserfora und dem Concordia-Tempel in Rom oder dem Kaisersaal von Side zumindest partiell eingeschränkt. Hier waren es vor allem die funktionalen Voraussetzungen, durch die die Aufmerksamkeit der Besucher vielfach in ganz andere Richtungen, auf die öffentlichen und sozialen Vorgänge in diesen Räumen, gelenkt wurde. Paul Zanker hat am Beispiel der Kaiserfora von Rom hervorgehoben, dass hier wohl einerseits vielfach nur eine allgemeine Atmosphäre von Bau- und Bildwerken erfahren wurde, andererseits häufig ganz selektiv 
einzelne Bildwerke wahrgenommen wurden, ohne dass dabei ein übergreifendes ,Programm‘ in den Blick kam. ${ }^{46}$

Diese starke Einschränkung der Sichtbarkeit und der Wahrnehmung hatte jedoch in keiner Weise Konsequenzen für den künstlerischen Rang und den thematischen Reichtum des Bauschmuckes. An den Skulpturen des Parthenon haben Generationen von Archäologen und Kunstkennern die superbe Qualität der plastischen Gestaltung gepriesen; der Fries und die Giebel haben eine Vielzahl von Deutungen hervorgerufen, die gerade in ihren Kontroversen die Komplexität der Bildthemen bezeugen.

Man darf diese scheinbar diskrepanten Phänomene nicht gegeneinander ausspielen. Die eingeschränkte Sichtbarkeit bedeutet nicht, dass komplexe Formen und Themen keine Rolle spielen; und die anspruchsvollen Formen und Themen bedeuten nicht, dass sie doch irgendwie aus der Nähe zu sehen gewesen sein müssen. Eine sinnvolle Erklärung muss die scheinbare Diskrepanz aufzulösen versuchen.

Die Frage kann hier nicht in der ganzen Tragweite untersucht werden. Deutlich ist aber, dass die traditionellen Ansätze einer semiotischen Kommunikationstheorie hier an Grenzen stoßen. Diese gehen in der Regel von einer Symmetrie der intensiven Produktion und der ebenso intensiven Rezeption von - in diesem Fall visuellem ,Sinn‘ aus: Die ,Sender‘ produzieren Bilder, die als autonome ,Zeichen` dichten ,Sinn“ transportieren, den die Empfänger als solchen zu verstehen suchen. Dies Modell erweist sich als zu einfach, offensichtlich sind die Phänomene vielschichtiger. Sie können aber in einem erweiterten Konzept der Kommunikation von visuellem Sinn erfasst werden. ${ }^{47}$

Die Bilder der antiken Bauwerke sind, bei aller potentiellen Komplexität, zunächst als ,ornamentale‘ Elemente auf die Architektur bezogen. An und mit den Bauwerken bilden sie, in dieser potentiellen Komplexität, einen ,Kosmos', in dem materieller, ideeller und ästhetischer ,Wert‘ in eins fallen: Der Begriff ,Kosmos‘ benennt grundsätzlich die normative Ordnung der menschlichen Kultur, von festlicher Kleidung und Schmückung der Menschen über ,richtige Verhaltensweisen bis zur geordneten Aufstellung von Heeren, dann aber auch die regelhafte Ordnung der Natur; speziell in der Architektur bezeichnet ,Kosmos' die dekorativen Elemente, von Friesen und Metopen-Triglyphen-Reihen über schmückende Standbilder bis zu ephemeren Girlanden. ${ }^{48}$ Die Betrachter sind zunächst dazu aufgerufen, diesen ,Kosmos ‘ als solchen wahrzunehmen, der in seiner Gesamtheit die Steigerung des kulturellen Wertes des Bauwerks leistet. Diese integrale Funktion des figürlichen Schmuckes ist der Funktion der intensiven Übermittlung von spezifischem ,Sinn` in den einzelnen Elementen

46 Zanker 1997.

47 Siehe dazu demnächst T. Hölscher 2018.

48 Zum Folgenden siehe ausgezeichnet Marconi 2004. Allgemein zum Begriff und Konzept des Kosmos: Casevitz 1989; Cartledge 1998; Finkelberg 1998; in diesem Band: Squire, 2, Anm. 3; Dietrich, 167-172; Barham. Zum Begriff des Kosmos in der Architektur: Hellmann 1992, 231-233. 
der Bildwerke übergeordnet. Das Sehen soll in dieser Abstufung der Stiftung von Sinn erfolgen: Die Sichtbarkeit im Einzelnen steht hinter der Wahrnehmung des Ganzen zurück.

Das bedeutet aber auf keinen Fall, dass die einzelnen Bilder auf die Schaffung von ästhetischer Kraft und thematischer Komplexität verzichten müssten. Im Gegenteil: Der ,Kosmos“ des Bauwerks als materielle, thematische und ästhetische ,Ordnung“ entsteht und besteht aus der Konstellation der einzelnen hochrangigen und komplexen Elemente: der kostbaren Materialien, der technisch perfekt geschnittenen Quadern und Säulen, und darüber hinaus der brillanten Ornamente und der suggestiv gestalteten Bilder. Ohne die Hochrangigkeit im Einzelnen würde auch das Ganze keinen kulturellen ,Wert“ erreichen.

Die Referenz des einzelnen Bildes auf die Architektur, in die die Bilder eingebunden sind, wird vielfach durch ,ornamentale‘ Gestaltung besonders hervorgehoben. Sie kann sich in forcierter Integration wie in Prinias, in stärkerer Autonomie wie am Parthenon, oder in ornamentaler Subordination wie in den späteren Girlanden-, Ranken-, Waffen- und Sakralgerät-Friesen zeigen.

Grundsätzlich aber besitzt der ,Wert“ des Dekors eine gewisse intrinsische Autonomie, die über die Sichtbarkeit hinaus reicht, jedenfalls nicht in der Wirkung der Sichtbarkeit aufgeht. Kosmos im Sinn von - materieller, thematischer, ästhetischer ,Ordnung' muss als solcher vollständig sein, gleich ob es sich um die Ordnung eines geschmückten Bauwerks, die Ordnung einer Polis oder die Ordnung der Welt und des Universums handelt. Der Mensch, der die Ordnung betrachtet und zu verstehen sucht, wird sie in der Regel nur unvollständig wahrnehmen können: Einerseits werden ihm bestimmte Teile verborgen bleiben, andererseits wird er bestimmte Teile nur unvollkommen und ungefähr wahrnehmen können. Das gilt für die entfernteren Zonen der Welt und des Universums, für die Randbereiche der Polis und der Bürgerschaft, wie für die schlecht oder nicht sichtbaren Teile einer Architektur. ${ }^{49}$

In allen diesen Fällen ist es aber entscheidend, dass der Betrachter die Gewissheit hat, dass die Ordnung zum einen tatsächlich vollständig ist, auch in den Bereichen, die ihm verborgen bleiben; und dass sie zum anderen in allen Teilen eine geordnete Form hat, auch dort, wo er es sie nur noch ungenau sehen kann. Das gilt auch für die Bild-Elemente der Architektur, die in der Höhe nur eingeschränkt sichtbar sind, wie für die, die an unzugänglichen Stellen verborgen sind.

Dies ist der eigentliche Sinn der Wahrnehmung: nicht reines Perzipieren, sondern das Gesehene ,für wahr nehmen‘ und sich selbst in die wahrgenommene Ordnung einzu-,ordnen'.

49 Zur (relativen) Autonomie der Bildwerke gegenüber den Betrachtern und ihren Voraussetzungen des Sehens siehe vorläufig T. Hölscher 2012. 


\section{Bibliographie}

Andronikos, M. (1989) Vergina: The Royal Tombs and the Ancient City. Athen.

Arrigoni, G. (1985) „Donne e sport nel mondo antico greco“, in Le donne in Grecia, hrsg. G. Arrigoni. Rom: 155-201.

Barlou, V. (2014) Die archaische Bildhauerkunst von Paros. Wiesbaden.

Beyer, I. (1976) Die Tempel von Dreros und Prinias A und die Chronologie der kretischen Kunst des 8. und 7. Jahrhunderts v. Chr. Freiburg.

Bielfeldt, R. (2012) „Polis made manifest: The physiognomy of the public in the Hellenistic city - with a case study of the agora of Priene", in Politische Kommunikation und öffentliche Meinung in der antiken Welt, hrsg. Chr. Kuhn. Stuttgart: 87-122.

Böhm, St. (1990) Die Nackte Göttin. Mainz.

Bol, P. C. (Hrsg.) (2002) Die Geschichte der antiken Bildhauerkunst. I: Frühgriechische Plastik. Mainz.

Bravi, A. (1998) „Tiberio e la collezione di opere d'arte dell’Aedes Concordiae Augustae“, Xenia Antiqua 7: 41-82.

Bravi, A. (2014) Griechische Kunstwerke im politischen Leben Roms und Konstantinopels. Berlin. Calame, C. (1977) Les chœurs de jeunes filles en Grèce ancienne. Rom.

Cartledge, P. (1998) „Introduction: Defining a kosmos“, in Kosmos. Essays in Order, Conflict and Community in Classical Athens, hrsg. P. Cartledge, P. Millett und S. von Reden. Cambridge: $1-12$.

Casevitz, M. (1989) „À la recherche du kosmos: là où tout n'est qu'ordre et beauté“, Le temps de la réflexion 10: 97-119.

Castriota, D. (1995) The Ara Pacis Augustae and the Imagery of Abundance in Late Greek and Early Roman Imperial Art. Princeton.

Celani, A. (1998) Opere d'arte greche nella Roma di Augusto. Napoli.

Coarelli, F. (1971-1972) „Il complesso pompeiano di Campo Marzio e la sua decorazione scultorea“, Rendiconti della Pontificia Accademia di Archeologia 44: 99-122.

Coarelli, F. (1988) Il sepolcro degli Scipioni a Roma. Rom.

D’Acunto, M. (1995) „I cavalieri di Prinias ed il tempio A“, Annali di Archeologia e di Storia Antica 2.1: 15-55.

Duplouy, A. (2006) Le prestige des élites: recherches sur les modes de reconnaissance sociale en Grèce entre les Xe et Ve siècles av. J.-C. Paris.

Eule, C. (2001) Hellenistische Bürgerinnen aus Kleinasien. Istanbul.

Finkelberg, A. (1998) „On the history of the Greek kosmos“, Harvard Studies in Classical Philology 98: 103-136.

Franssen, J. (2011) Votiv und Repräsentation. Statuarische Weihungen archaischer Zeit aus Samos und Attika. Heidelberg.

Fredricksmeyer, E. A. (1997) „The origin of Alexander's royal insignia“, Transactions of the American Philological Association 127: 97-109.

Froning, H. (1981) Marmor-Schmuckreliefs mit griechischen Mythen im 1. Jahrhundert v. Chr. Mainz.

Fuchs, M. (1981-1982) „Eine Musengruppe aus dem Pompejus-Theater“, Mitteilungen des Deutschen Archäologischen Instituts Rom 89: 69-80.

Fuchs, M. (1987) Untersuchungen zur Ausstattung römischer Theater in Italien und den Westprovinzen des Imperium Romanum. Mainz.

Galinier, M. (2007) La colonne Trajane et les forums impériaux. Paris.

Giuliani, L. (1986) Bildnis und Botschaft. Hermeneutische Untersuchungen zur Bildniskunst der römischen Republik. Frankfurt am Main.

Graeve, V. von (1970) Der Alexandersarkophag und seine Werkstatt. Berlin. 
Griesbach, J. (2013) „Zur Topographie hellenistischer ,Ehrenstatuen“ auf Delos“, in Roman Power and Greek Sanctuaries: Forms of Interaction and Communication, hrsg. M. Galli. Athen: 83-124.

Grunauer, P. (1974) „Der Westgiebel des Zeustempels von Olympia - Die Münchner Rekonstruktion, Aufbau und Ergebnisse“, Jahrbuch des Deutschen Archäologischen Instituts 89: 1-51.

Grüßinger, R. (2001) Dekorative Architekturfriese in Rom und Latium. Online verfügbar unter: http://d-nb.info/1005585121/34 (zuletzt heruntergeladen am 05. 04. 2017).

Hellmann, M.-Chr. (1992) Recherches sur le vocabulaire de l'architecture grecque d'après les inscriptions de Délos. Paris.

Herrmann, H.-V. (1987) „Die Kontroverse um die Mittelgruppe des Ostgiebels“, in Die OlympiaSkulpturen, hrsg. H.-V. Herrmann. Darmstadt: 125-148.

Hesberg, H. von (1981) „Girlandenschmuck der republikanischen Zeit in Mittelitalien“, Mitteilungen des Deutschen Archäologischen Instituts Rom 88: 201-245.

Himmelmann, N. (1968) Über einige gegenständliche Bedeutungsmöglichkeiten des frühgriechischen Ornaments. Wiesbaden.

Hölscher, F. (1972) Die Bedeutung archaischer Tierkampfbilder. Würzburg.

Hölscher, F. (2014) „Gottheit und Bild - Gottheit im Bild“, in Ding und Mensch in der Antike. Gegenwart und Vergegenwärtigung, hrsg. R. Bielfeldt. Heidelberg: 239-256.

Hölscher, T. (1989) „Griechische Bilder für den römischen Senat“, in Festschrift für N. Himmelmann, hrsg. H.-U. Cain. Mainz: 327-333.

Hölscher, T. (1994) „Hellenistische Kunst und römische Aristokratie“, in Das Wrack. Der antike Schiffsfund von Mahdia, hrsg. G. Hellenkemper-Salies. Köln: 875-888.

Hölscher, T. (1999) „Immagini mitologiche e valori sociali nella Grecia antica“, in Im Spiegel des Mythos. Bilderwelt und Lebenswelt, hrsg. F. de Angelis und S. Muth. Wiesbaden: 11-30.

Hölscher, T. (2000) „Einführung“, in Gegenwelten zu den Kulturen Griechenlands und Roms in der Antike, hrsg. T. Hölscher. München: 9-18.

Hölscher, T. (2002) „Rituelle Räume und politische Denkmäler im Heiligtum von Olympia“, in Olympia 1875-2000. 125 Jahre deutsche Ausgrabungen, hrsg. H. Kyrieleis. Mainz: 331-345.

Hölscher, T. (2009) „Architectural sculpture: Messages? Programs? Towards rehabilitating the notion of , decoration “', in Structure, Image, Ornament, hrsg. P. Schultz und R. Von den Hoff. Oxford: 54-69.

Hölscher, T. (2012) „Bilderwelt, Lebensordnung und die Rolle des Betrachters im antiken Griechenland“", in Raum Bild Handlung. Perspektiven der Archäologie, hrsg. O. Dally, S. Moraw, und H. Ziemssen. Berlin: 19-44.

Hölscher, T. (2015a) La vie des images grecques: sociétés de statues, rôles des artistes, notions esthétiques dans l'art grec ancien. Paris.

Hölscher, T. (2015b) „Die Entstehung der griechischen Polisgemeinschaft im Bild: Lebende, Vorfahren, Götter“, in Leibhafte Kunst. Statuen und kulturelle Identität, hrsg. D. Boschung und Chr. Vorster. Paderborn: 13-53.

Hölscher, T. (2015c) „Noch einmal rechts und links am Zeustempel von Olympia“, in New Approaches to the Temple of Zeus at Olympia, hrsg. A. Patay-Horváth. Newcastle-upon-Tyne: 90-93.

Hölscher, T. (2018) Visual Power in Ancient Greece and Rome. Berkeley.

Honroth, M. (1971) Stadtrömische Girlanden. Wien.

Hupfloher, A. (2012) „Heraion und Herakult im kaiserzeitlichen Olympia“, Archiv für Religionsgeschichte 13: 225-252.

Inan, J. (1975) Roman Sculpture in Side. Ankara.

Kellum, B. (1990) „The city adorned: Programmatic display at the Aedes Concordiae Augustae“, in Between Republic and Empire, hrsg. K. Raaflaub und K. Toher. Berkeley: 276-307.

Kottaridi, A. (2013) Aigai: The Royal Metropolis of the Macedonians. Athen. 
Kreilinger, U. (2007) Anständige Nacktheit. Rahden.

Krumeich, R. (2008) „Vom Haus der Gottheit zum Museum? Zu Ausstattung und Funktion des Heraion von Olympia und des Athenatempels von Lindos“, Antike Kunst 51: 73-94.

Kyrieleis, H. (2012/2013) „Pelops, Herakles, Theseus: Zur Interpretation der Skulpturen des Zeustempels von Olympia“, Jahrbuch des Deutschen Archäologischen Instituts 127/128: 51-124.

La Rocca, E. (1983) Ara Pacis Augustae. Rom.

Linfert, A. (1979) [Rezension zu Inan 1975,] Bonner Jahrbücher des Rheinischen Landesmuseums Bonn 179: 780-785.

Linfert, A. (1995) „Die Skulpturen des Kaisersaales von Side (Pamphylien)“, in Was ist eigentlich Provinz? Zur Beschreibung eines Bewusstseins, hrsg. H. von Hesberg: 153-169.

Ma, J. (2013) Statues and Cities: Honorific Portraits and Civic Identity in the Hellenistic World. Oxford.

Manderscheid, H. (1981) Die Skulpturenausstattung kaiserzeitlicher Thermenanlagen. Berlin.

Mansel, A. F. (1963) Die Ruinen von Side. Berlin.

Marconi, Cl. (2004) „Kosmos: The imagery of the Archaic Greek temple“, RES: Anthropology and Aesthetics 45: 211-224.

Marconi, Cl. (2009) „The Parthenon frieze: Degrees of visibility“, RES: Anthropology and Aesthetics 55-56: 156-173.

Marinatos, N. (2000) The Goddess and the Warrior: The Naked Goddess and Mistress of Animals in Early Greek Religion. London.

Maschek, D. (2012) Rationes decoris. Aufkommen und Verbreitung dorischer Friese in der mittelitalischen Architektur des 2. und 1. Jahrhunderts v. Chr. Wien.

Millar, F. (1977) The Emperor in the Roman World. Ithaca.

Mitropoulou, E. (1993) „The origin and significance of the Vergina symbol“, in Archaia Makedonia 5: 843-958.

Mlasowski, A. (2010) Ara Pacis. Mainz.

Möbius, H. (1929) Die Ornamente der griechischen Grabstelen. Berlin.

Moreno, P. (1987) Pittura greca. Mailand.

Müller, F. M. (2009) „Der Stern von Vergina: Vom Emblem der makedonischen Königsdynastie zum nationalen Symbol Griechenlands“, in Bildmagie und Brunnensturz. Visuelle Rhetorik von der Klassischen Antike bis zur aktuellen medialen Kriegsberichterstattung, hrsg. E. Walde. Innsbruck: 364-380.

Niemeyer, H.-G. (1996) Über den Sinn griechischer Standbilder. Göttingen.

Olivito, R. (2013) Il foro nell'atrio: immagini di architetture, scene di vita e di mercato nel fregio di Iulia Felix (Pompei, II, 4, 3). Bari.

Papini, M. (2004) Antichi volti della Repubblica: la ritrattistica in Italia centrale tra IV e II secolo AC. Rom.

Patay-Horváth, A. (2007) „Zur Rekonstruktion und Interpretation des Ostgiebels des Zeustempels von Olympia“, Jahrbuch des Deutschen Archäologischen Instituts 122: 161-206.

Patay-Horváth, A. (Hrsg.) (2015) New Approaches to the Temple of Zeus at Olympia. Newcastle-uponTyne.

Polito, E. (1998) Fulgentibus armis. Rom.

Raeck, W. (1995) „Der mehrfache Apollodoros: Zur Präsenz des Bürgers im hellenistischen Stadtbild am Beispiel Prienes“, in Stadtbild und Bürgerbild im Hellenismus, hrsg. M. Wörrle und P. Zanker. München: 231-240.

Ridgway, B. S. (1999) Prayers in Stone: Greek Architectural Sculpture ca. 600 - 100 B.C.E. Berkeley.

Rolley, Cl. (2003) La tombe princière de Vix. Paris.

Rossini, 0. (2006) Ara Pacis. Rom.

Rouveret, A. (1989) Histoire et imagination de la peinture ancienne: Ve siècle av. J.C. - ler siècle ap. J.C. Paris. 
Sauron, G. (1987) „Le complexe pompéien du Champ de Mars“, in L'Urbs: espace urbain et histoire

(ler siècle av. J.C. - Ille siècle ap. J.C. Collection de l'École Française de Rome 98. Rom: 457-473.

Sauron, G. (2000) L'histoire végétalisée: ornement et politique à Rome. Paris.

Sauron, G. (2013) Augusto e Virgilio: la rivoluzione artistica dell'Occidente e l'Ara Pacis. Mailand.

Schäfer, Th. (1989) Imperii insignia. Sella curulis und fasces. Mainz.

Scheibler, I. (1994) Griechische Malerei der Antike. München.

Schmidt, E. (1982) Geschichte der Karyatide. Würzburg.

Schörner, G. (1995) Römische Rankenfriese. Mainz.

Sehlmeyer, M. (1999) Stadtrömische Ehrenstatuen der republikanischen Zeit. Stuttgart.

Settis, S. (1992) „Die Trajanssäule: Der Kaiser und sein Publikum“, in Die Lesbarkeit der Kunst. Zur Geistes-Gegenwart der Ikonologie, hrsg. A. Beyer. Berlin: 40-52.

Simon, E. (1967) Ara Pacis Augustae. Tübingen.

Simon, E. (1968) „Zu den Giebeln des Zeustempels von Olympia“, Mitteilungen des Deutschen Archäologischen Instituts Athen 83: 147-166.

Simon, E. (2010) Ara Pacis Augustae. Dettelbach.

Spannagel, M. (1999) Exemplaria Principis. Heidelberg.

Stähli, A. (2002) „Bild und Bildakte in der griechischen Antike“, in Quel corps? Eine Frage der Repräsentation, hrsg. H. Belting, D. Kamper und M. Schulz. München: 67-84.

Steuben, H. von (1979) Der Kanon des Polyklet. Tübingen.

Stewart, A. (1997) Art, Desire and the Body in Ancient Greece. Cambridge.

Sydow, W. von (1974) „Die Grabexedra eines römischen Feldherrn“, Jahrbuch des Deutschen Archäologischen Instituts 89: 187-216.

Vernant, J.-P. (1985) Mythe et pensée chez les Grecs: études de psychologie historique. Paris.

Veyne, P. (1988) „Conduites sans croyance et œuvres d'art sans spectateurs“, Diogène 143: 3-22.

Veyne, P. (1990) „Propagande expression roi, image idole oracle“, L’Homme 114, 30(2): 7-26.

Walter, U. (2004) Memoria und res publica. Zur Geschichtskultur im republikanischen Rom. Frankfurt am Main.

Watzinger, C. (1905) Griechische Holzsarkophage aus der Zeit Alexanders des Großen. Leipzig.

Webb, P. A. (1996) Hellenistic Architectural Sculpture. Madison.

Winkler- Horaček, L. (2015) Monster in der frühgriechischen Kunst. Die Überwindung des Unfassbaren. Berlin.

Zanker, P. (1968) Forum Augustum. Das Bildprogramm. Tübingen.

Zanker, P. (1997) „In search of the Roman viewer“, in The Interpretation of Architectural Sculpture in Greece and Rome, hrsg. D. Buitron-Oliver. London: 179-191.

\section{Abbildungsnachweise}

Abb. 2.1: Repro aus Kottaridi 2013, 152.

Abb. 2.2: Repro aus Rolley 2003, Taf. 4.

Abb. 2.3a-c: Repro aus Beyer 1976, Taf. 13, 21 und 24.

Abb. 2.4: Repro aus Kyrieleis 2012/2013, 54, Abb. 1 b.

Abb. 2.5: () Fotoarchiv Hölscher. Foto Dieter Johannes.

Abb. 2.6: Repro aus von Steuben 1973, 13, Abb. 1.

Abb. 2.7: ( ) Photothek, Institut für Klassische Archäologie Heidelberg.

Abb. 2.8a: Repro aus Mansel 1963, Abb. 90.

Abb. 2.8b: Verteilung der gefundenen Skulpturen nach T. Hölscher. 